\title{
Die Sprache des byzantinischen Alexandergedichtes.
}

Vorbemerkung. Der Text des Gedichtes, dessen Sprache im Folgenden einer Untersuchung unterzogen werden soll, ist überliefert in einer Papierhandschrift in $4^{0}$ aus dem 14. Jahrhundert, die sich in der Markusbibliothek in Venedig befindet (vgl. Berger de Xivrey, Notices et extraits XIII p. 203; Zacher, Pseudokall. S. 25 f., 28 f.; Kapp in der gleich anzuführenden Abhandlung S. 1-3). Der vollständige Text dieses aus 6118 politischen Versen bèstehenden Gedichtes ist zuerst herausgegeben aus dem Nachlasse W. Wagners von D. Bikélas in Trois poèmes grecs du moyen-âge inédits. Recueillis par le feu prof. W. W. Berlin, Calvary, 1881 S. 56-241. Einzelne Teile (im ganzen 470 Verse, 1-199, 5569-5813, 6093-6118) und die grölste Anzahl der Lemmata oder Inhaltsangaben waren vorher veröffentlicht von St. Kapp, Mitteilungen aus zwei griech. Hss zur Gesch. der Alexandersage im Mittelalter, Pr. des Obergymn. Wien IX, 1872, S. 1-38. Endlich ist der erste Teil (v. $1-772$ bei Wagner $=817$ Verse bei Legrand, da dieser die Lemmata fortlaufend mitzählt) herausgegeben von Legrand in Bibliothèque grecque vulgaire publiée par E. L. t. II, Paris 1881. Die weitere Publikation ist $t_{2}$ wie eine Notiz Legrands angiebt (p. LIX), unterblieben, weil inzwischen die Wagnersche Ausgabe erschienen war. Das Gedicht, dessen Verf. unbekannt ist, ist verfafst zwischen den Jahren 1200 und 1350, da einerseits die Chronik des Zonaras benutzt ist, und die Abschrift aus dem Jahre 1388 stammt, wie die Unterschrift am Schlusse des Gedichtes beweist:

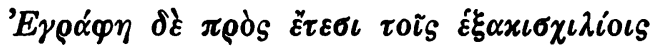

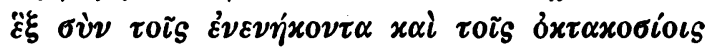

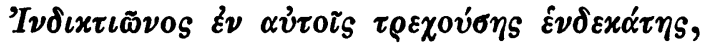

d. h. im Jahre d. W. $6896=1388$ u. Ä. Dafs dies Jahr nicht das Jahr der Abfassung ist, vielmehr vermutlich diese Abschrift schon nicht vom Original genommen ist, beweisen mancherlei Fehler, die sich eingeschlichen haben, z. B. das Eindringen einzelner Lemmata in den Text und die Auslassung einzelner Verse. 
Der Titel des Gedichtes, welches eine Erzählung der Alexandergeschichte in der sagenhaften Form des Pseudo-Kallisthenes enthält, ist

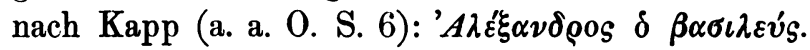

\section{Formenlehre.}

\section{Accent.}

In der Accentuierung ${ }^{1}$ ) finden sich einige, wenn auch nicht gerade zahlreiche Abweichungen von der früher üblichen Betonung, die teils mit der zur Zeit des Verf. üblichen Betonung in der Volkssprache übereinstimmen, teilweise auch nur dem Bedürfnis des Verses ihren Ursprung verdanken. $\mathrm{Zu}$ der ersteren Art sind wohl jedenfalls $\mathrm{zu}$ rechnen die Betonungen des Genetivs Pluralis $\beta$ or $\alpha^{\prime} \nu \omega \nu$ (253), $\pi \iota \nu \varepsilon x \chi v$ $\tau \omega \nu$ (3471), $\vartheta \eta \lambda \varepsilon i \omega \nu$ (4937), $\tau \varepsilon i \chi \omega \nu$ (2226); auch die Betonung $\delta \varepsilon \nu$ $\delta \varrho \tilde{\nu} \nu$ von dem im vulgärgriechischen gewöhnlichen $\delta \varepsilon v \delta \rho o ́ v$ findet sich (viermal: 3334. 3633. 4727. 4782), während der Verf. sonst immer

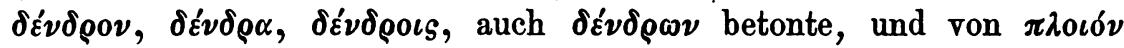
$\pi \lambda_{0} \tilde{\omega} \boldsymbol{\nu}(1204,6)$. Ebenso gehören hierher anscheinend die Accente

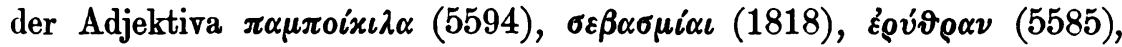

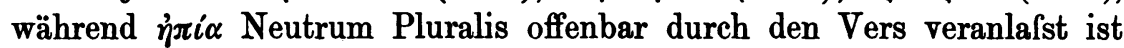
(4300). Ferner gehört hierher die Betonung der Verbalformen $\pi \varepsilon \tau \alpha \dot{\sigma} \sigma \alpha \iota$ (364), $\lambda \alpha^{\prime} \beta \varepsilon$ (344. 4899. 5059. 5183), i' $\delta \varepsilon$ (1830. 3682), während $\varepsilon i \pi \varepsilon ́$ (974) und $\dot{\varepsilon} \lambda \vartheta \varepsilon^{\prime}$ (2101) die früher übliche Accentuation bewahrt haben;

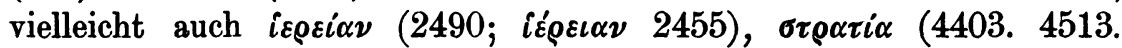

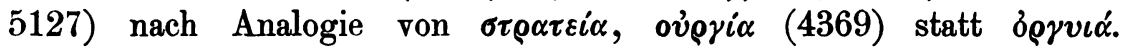
Durchaus der vulgären Betonung entspricht endlich die Accentuation $\alpha v^{\tau} \tau$ s u. s. w., die zweimal in dem Wagnerschen Texte erscheint:

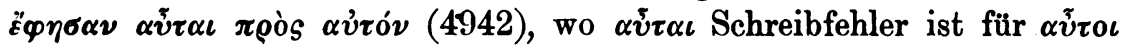

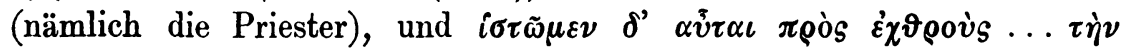
$\mu \alpha ́ \chi \eta \nu$ (5523); ebenso ist aber wohl sicher zu schreiben v. 2987: $\ddot{\varepsilon} \nu$ -

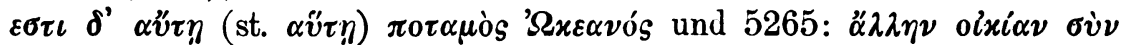

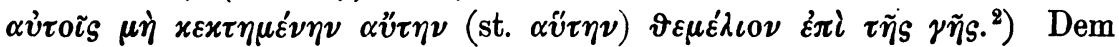

1) Vgl. Krumbacher, Beitr. z. einer Gesch. d. griech. Sprache, in Kuhns Ztschr. 27, $522 \mathrm{ff}$; Hatzidakis, Einleitung in d. neugriech. Grammatik, Exkurs X S. $418 \mathrm{ff}$.

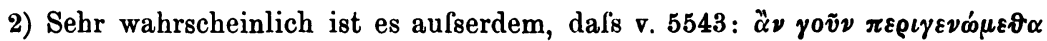

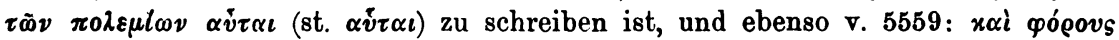

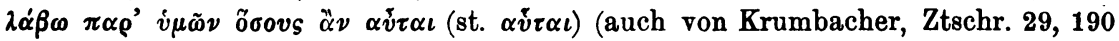

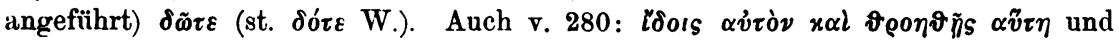

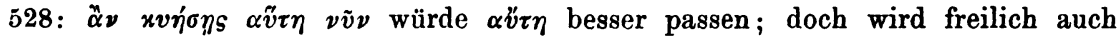

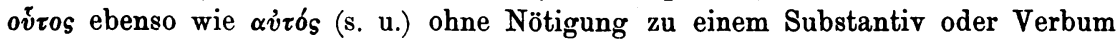
hinzugefügt, z. B. ganz übereinstimmend mit der ersten Stelle v. 4511: $\mu \grave{\eta}$ 
Versbedürfnis werden dagegen vermutlich die Änderungen des Accents

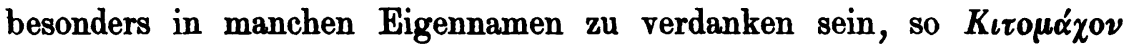

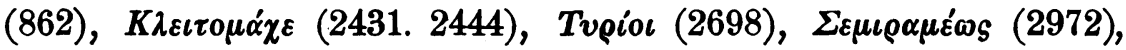
'A $A \varepsilon \xi \alpha \nu \delta \rho \varepsilon i \alpha \nu$ (1323. 5424. 6102, 3, 6); und ebenso bei den Wörtern

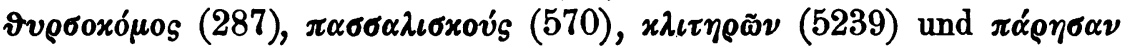
(1476. 4783. 4891. 5588; 5245 ist $\pi \alpha \varrho \tilde{\eta} \sigma \alpha \nu$ zu accentuieren). Jedenfalls mufs an den angeführten Stellen der angegebene Accent des Verses wegen stehen, und dafs man aus diesem Grunde einfach den Accent

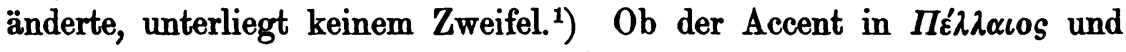
É $\rho_{\gamma} \delta_{\delta} \varepsilon_{S}$ mit Absicht oder nur aus Nachlässigkeit gesetzt ist, wage ich nicht $\mathrm{zu}$ entscheiden.

\section{Deklination.}

Wenn die Deklinationsformen auch gröfstenteils mit denen der klassischen Sprache übereinstimmen, so begegnen doch auch solche, die beweisen, dafs der Verf. sich dem Einflufs der Volkssprache nicht hat entziehen können. So treffen wir bei ihm sowohl Formen, die einen gleichsilbigen statt eines ungleichsilbigen Nominativs ${ }^{2}$ ) voraussetzen, wie auch andere vulgärgriechische Deklinationsformen, nämlich:

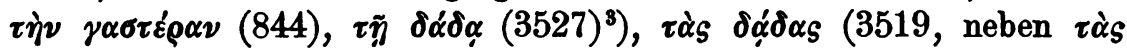

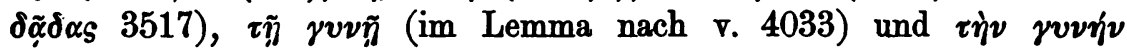

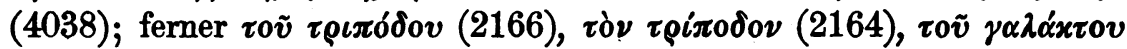

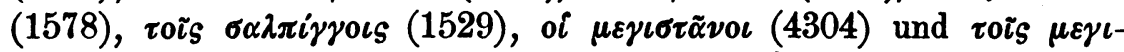

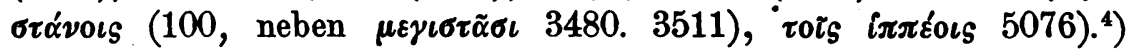
Dahin wird auch gehören der Accusativ Pluralis $\pi \eta^{\prime} \chi \alpha_{S}(5752.5780)^{5}$ )

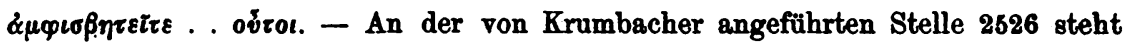

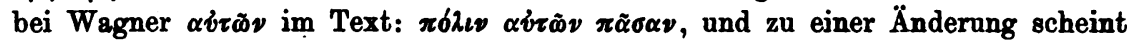
kein Grund vorhanden. Des Verses wegen braucht jedenfalls nicht geändert zu werden; denn wenn auch im allgemeinen die drittvorletzte Silbe des Verses von unserm Verf. nicht accentuiert wird, so kommen doch an dieser Stelle sowohl die oxytonierten und zirkumflektierten Formen des Artikels wie auch einsilbige

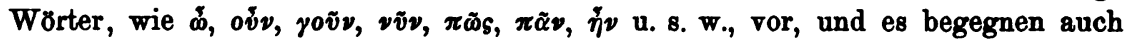
andere zirkumflektierte Formen - im ganzen in den 6118 Versen $38 \mathrm{mal}$ - an

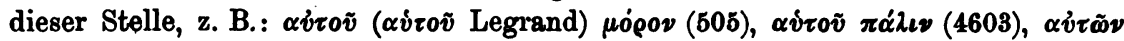
$\tilde{\alpha} \pi \alpha \nu$ (2368), aufserdem $\dot{\eta} \mu \tilde{\omega} \nu, i \dot{\nu} \tilde{\nu} \nu, \hat{\eta} \mu \tilde{\nu} \nu, \dot{v} \mu \tilde{i} \nu$ (z. B. 5522. 2884. 3201. 2533), $\pi 0 \lambda \lambda \tilde{\omega}, \vartheta \varepsilon \tilde{\nu}$ u. 8. w.

1) Hatzidakis a. a. O. S. 433, vgl. auch Henrichsen, Ưb. d. sogen. polit. Verse b. den Griechen, übers. v. Friedrichsen, S. 55 f.

2) Krumbaoher in Kuhns Ztschr. 27, 534. Hatzidakis, Einl. 54. 80. 379. Vgl. Mullach, Gr. d. griech. Vulgärsprache $160 \mathrm{ff}$.

3) Utber das $\alpha$ im Dativ s. Hatzidakis, Einl. S. 76 ff.

4) Vgl. Hatzidakis a. a. O. S. 378.

5) Über $\pi \tilde{\eta} \chi v_{\S}$ Hatzidakis, Z. Gesch. des Mittel- u. Neugriech., Kuhns Ztschr. 30, 139, A. 1, vgl. Lobeck zu Phryn. p. 246. 
zu $\pi \tilde{\eta} \chi v \varsigma$, dessen Formen im Genetiv Pluralis $\pi \dot{\eta} \chi \varepsilon \omega \nu$ (z. B. 4669) und $\pi \eta \chi \tilde{\omega} \nu$ (z. B. 5776), im Dativ $\pi \eta \dot{\eta} \chi \varepsilon \sigma \iota$ lauten; und dazu wird auch zu rechnen sein der Genetiv und Dativ Pluralis $\pi \alpha \rho \delta \alpha ́ \lambda \omega \nu$ (4942) und

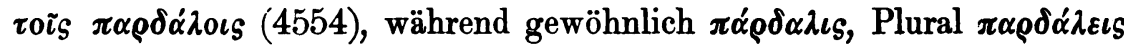

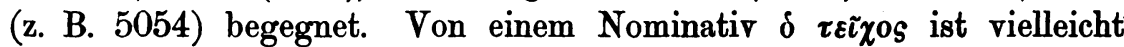

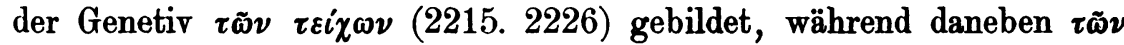

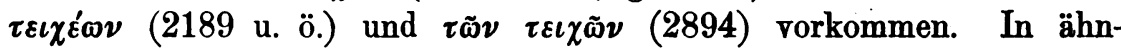
licher Weise finden wir neben den früher gebräuchlichen Accusativen $\nu \alpha \tilde{v} \nu$ und $\nu \alpha \tilde{v} S$ auch $\nu \tilde{\eta} \alpha(797), \nu \tilde{\eta} \alpha S(1184$ u. $\ddot{0}$.) und den Genetiv $\nu \eta \tilde{\omega} \nu$ (2897). ${ }^{1}$ ) Als Dativ Pluralis von $\tau \rho \iota \eta^{\prime} \eta_{S}$ erscheint $\tau \alpha \tau_{S} \tau \rho \iota \eta \dot{\rho} \alpha \iota s$

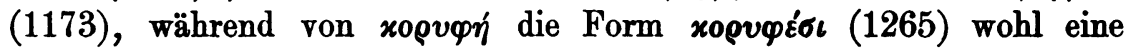
willkürlich vom Verf. gebildete Form ist. Allgemein angenommen waren dagegen schon seit verhältnismäfsig früher Zeit die Genetive und Vokative auf $-\eta$ (bezw. $-\alpha$ ) von Wörtern, besonders Eigennamen, auf $-\eta s$ (bezw. $-\alpha_{S}$ ) im Nominativ ${ }^{2}$ ); so begegnen hier $K \alpha \nu \delta \alpha v \lambda_{\eta}\left(5100 .^{3}\right)$

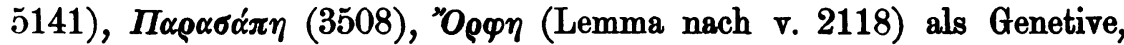

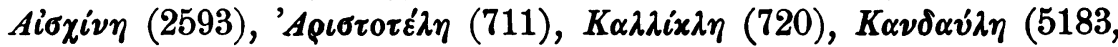

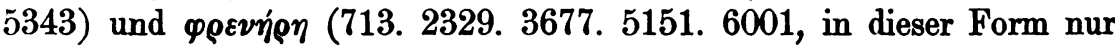
von' Alexander gesagt) als Vokative. Der Volkssprache gehören ferner jedenfalls an der Vokativ Sing. $\pi \alpha \nu v \pi \varepsilon ́\left(\gamma \lambda v x \varepsilon\right.$ (5889) und $\pi \delta \lambda_{\iota s}(2449)$,

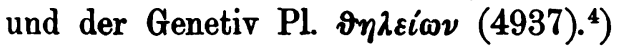

Abweichungen im Genus ${ }^{5}$ ) der Substantive begegnen nur ganz

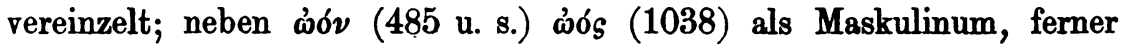

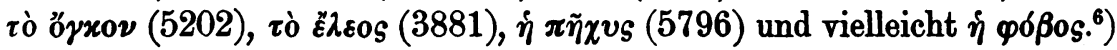

In der Deklination der Eigennamen treten ähnliche Erscheinungen uns entgegen, aber noch mehr Willkür des Verf., wie es scheint. Besonders interessant in dieser Beziehung ist die Deklination von ' $H \rho \alpha-$

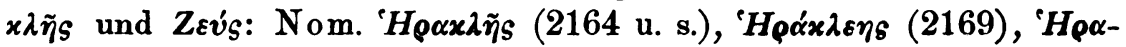

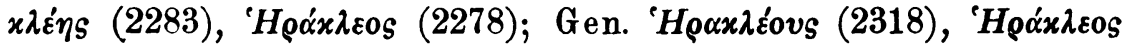

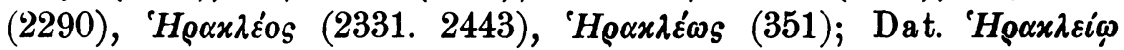

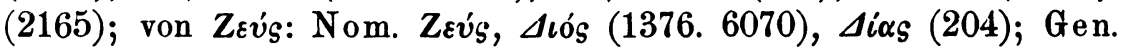

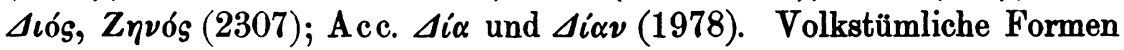
begegnen neben den schon besprochenen Genetiven und Vokativen auf

1) Vgl. Lobeck, Phryn. p. 170 . Hatzidakis, Einl. S. 16.

2) Hatzidakis, Z. neugriech. Dekl., Kuhns Ztschr. 32, 425; Einl. S. 16 f. 380 f.

3) Das $\iota$ subscriptum im Text bei Wagner ist zu tilgen, da der Verf. sonst

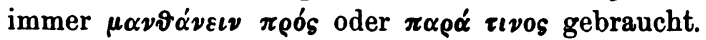

4) Hatzidakis, Einl. S. 381.

5) Uber Genuswechsel vgl. Hatzidakis, Einl. S. $354 \mathrm{ff}$. hier $\tau o ́ v$.

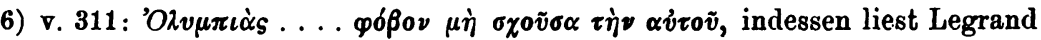




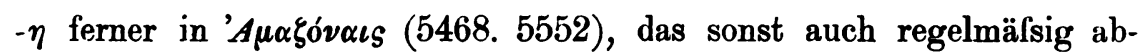
gewandelt wird und von dem auch eine Nebenform 'A $A \alpha \xi o v i$ S (z. B. 5491. 5541) vorkommt, und in 'A Nominativ 'A $\mu \varphi\left(x \tau v\right.$ oves ${ }^{1}$ ) (2735). Der Flufsname Tigris lautet im

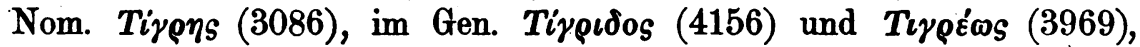

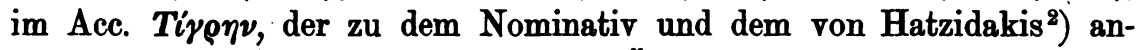

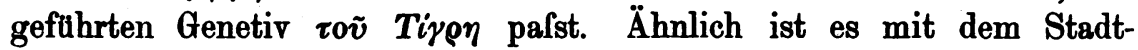
namen Memphis: im Acc. findet sich Mérqıv (1480. 6078), im Nom.

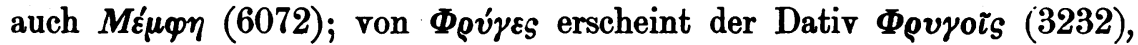

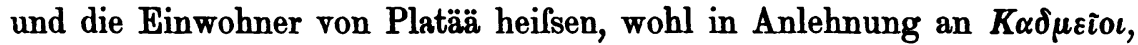
$\Pi \lambda \alpha \tau \varepsilon i o$, wie der Genetiv $\Pi \lambda \alpha \tau \varepsilon i \omega \nu$ (2465. 2611) zeigt, während umgekehrt für das gewöhnlich gebrauchte 'Ivdoi dreimal (4686. 4916. 4934) die Form 'I $\nu \delta \varepsilon \tilde{i}_{S}$ angewandt wird. Auf der Ausstofsung einer Silbe $^{3}$ ) beruht der Genetiv Singularis $\Pi \varepsilon \iota \varepsilon^{\prime} \omega s$ (2633), und die Formen

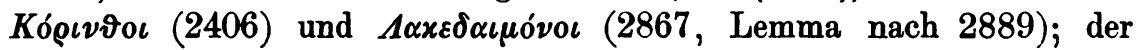

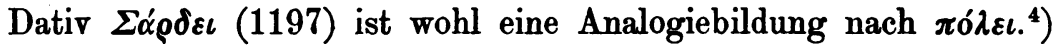

Aufser diesen an die Volkssprache sich anlehnenden Formen der Deklination zeigt der Verf. nun aber auch Freiheit und Willkür in der

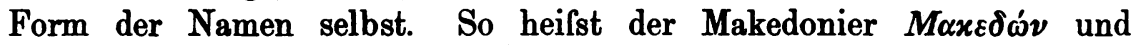

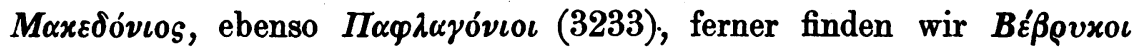

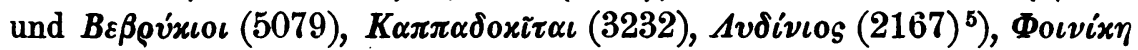
(1603). Auch in den Personennamen begegnet solche Willkür, und bisweilen werden sogar Buchstaben oder Silben, vermutlich nur dem

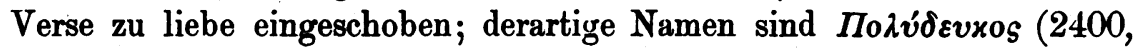

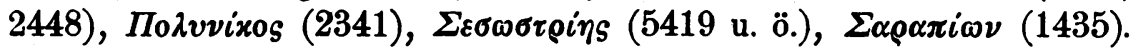

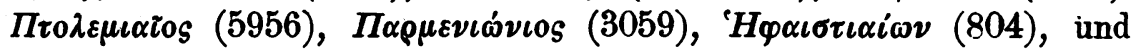

1) In der Hs scheint stets 'A wohl nur auf einem Versehen des Abschreibers, und es ist überall zu bessern.

2) Z. neugriech. Dekl., a. a. O. S. 425.

3) Hatzidakis, Einl. S. 336. Vermutlich ist eine ähnliche Ausstofsung auch

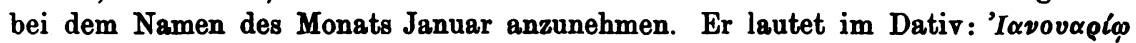
(6110), i. 1385 dagegen steht lovvvapiov. Da nun der Ubergang des u-Lautes auf die erste Silbe schwerlich möglich, das Schwinden des u-Lautes vor $\alpha$ dagegen gerade bei 'I $\alpha$ vovóplos belegt ist (Hatzidakis, Z. Vokalism. d. Neugr., Kuhns Ztschr. 30, 382, vgl. dens., Einl. S. 337), so wird als richtige Form wohl 'I $\alpha v \alpha \rho i o v$ herzustellen sein.

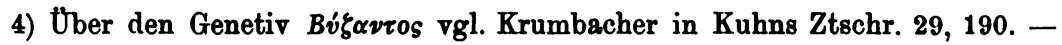

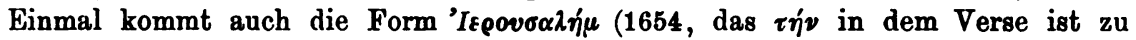
streichen) vor, wo das $I$ konsonantisch zu sprechen ist, vgl. Hatzidakis, Einl.

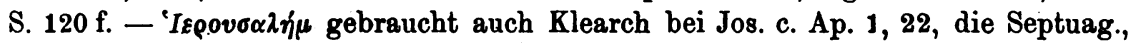
Theodor. i. d. Lobrede auf d. heil. Theodos. (Usener p. 87, 23).

5) In dem Verse ist statt $\Phi_{0 i} \beta_{0 s} z u$ lesen Kpoíos. 
für $\Phi i \lambda \iota \pi \pi 0 s$ eine Art Patronymikon $\Phi \iota \lambda \iota \pi \pi \iota \alpha ́ \delta \eta s$ (einmal im Acc.

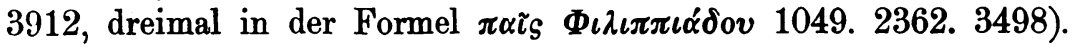

In betreff der Adjektiva sind die hauptsächlichsten Abweichungen in der Deklination schon angegeben. Hier ist noch zu bemerken, dafs neben der Nominativform $\mu \varepsilon \dot{\gamma} \alpha s$ einmal auch $\mu \varepsilon \gamma \alpha$ ádos (221) sich findet,

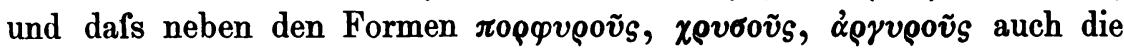
unkontrahierten vorkommen (z. B. 1892.1249 u. s.) und aufserdem

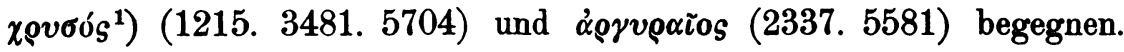
Ferner erscheint auch die volkstümliche Bildung auf -Eivos ${ }^{2}$ ) in $\pi 00$ -

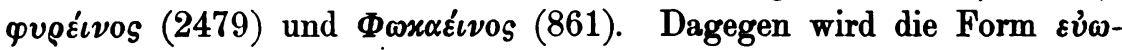
vúucos (4776) wohl der Willkür des Verf., des' Verses wegen, seine Entstehung rerdanken.

In der Komparation erscheinen die ja schon seit dem 1. Jahrhundert gebräuchlichen Formen $\tau \alpha^{\prime} \chi \iota \nu \nu$ und $\tau \alpha \chi v v_{\tau} \alpha \tau \alpha$ (als Adjektiv nur einmal 1274) sehr häufig; im übrigen sind die abweichenden Formen schon von Krumbacher angeführt worden ${ }^{3}$ ), sodafs ich von einer besonderen Aufzählung hier Abstand nehmen kann.

\section{Konjugation.}

Zunächst werden die Endungen und Vokale des Perfekts, Aorists und Imperfekts mit einander vertauscht. ${ }^{4}$ ) Demnach finden wir $\varepsilon i \lambda \eta$ -

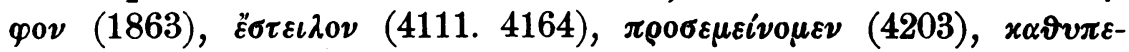
$\tau \alpha^{\prime} \xi \alpha \sigma \iota$ (4544); aus einer ähnlichen Vermischung der Formen des starken und schwachen ${ }^{5}$ ), wie des aktiven und passiven Aorists stammen die

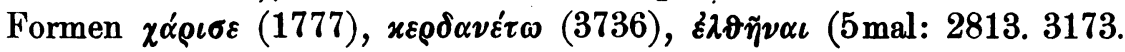

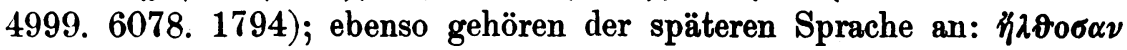

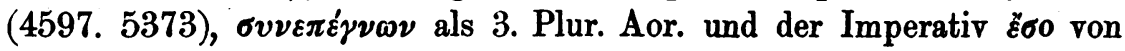
हiva८ (362). ${ }^{\circ}$ )

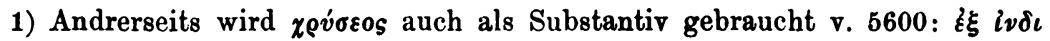

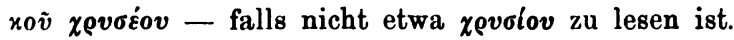

2) Hatzidakis, Einl. S. 181.

3) In Kuhns Ztschr. 29, 191. Ich füge hinzu $\pi \alpha \mu \mu \varepsilon \gamma \varepsilon \vartheta \dot{\varepsilon} \sigma \tau \alpha \tau o$ (597), $\pi \rho \circ \sigma \mu \varepsilon-$ $\gamma \varepsilon \vartheta \dot{\sigma} \sigma \tau \alpha \tau o s ~(403), \pi \lambda \eta \sigma\llcorner\varepsilon \dot{\sigma \varepsilon \rho o \nu ~(2010 ; ~ v g l . ~ S c h m i d, ~ A t t . ~ 1, ~ 195) ; ~ f e r n e r ~ d i e ~ K o m-~}$

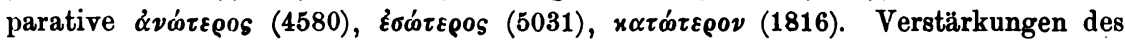
Superlativs finden sich aufser den beiden von Krumbacher angeführten noch:

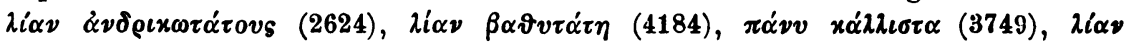

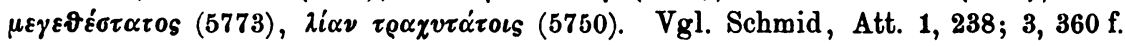

4) Hatzidakis, Einl. S. 112. 186.

5) Ders. S. 187. 192. Lobeck, Phryn. p. 349. Winer, Gr. S. 73. Mullach, Gr. S. 226.

6) Vgl. Mullach, S. 282. Schmid, Att. 3, 40. Vielleicht ist auch v. 3883 statt $\xi_{\sigma} \eta$ zu lesen $\boldsymbol{\varepsilon}_{\sigma o}$, da vorher und nachher lauter Imperative stehen: $\dot{\alpha} \boldsymbol{\nu} \alpha \dot{\alpha} \boldsymbol{\alpha} \alpha$, $\beta \alpha \sigma i \lambda \varepsilon v \varepsilon,\left(u^{\prime} \sigma o\right), \delta \varepsilon^{\prime} \xi \alpha \iota, \varepsilon^{\prime} \chi \varepsilon$. 
Auf einzelne Fälle beschränken sich auch die Abweichungen im Gebrauch des Augments bezw. der Reduplikation. ${ }^{1}$ ) Ohne Augment

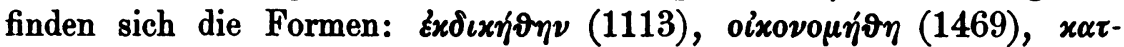

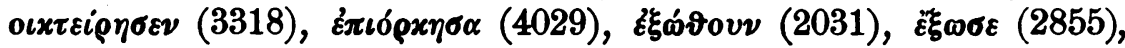

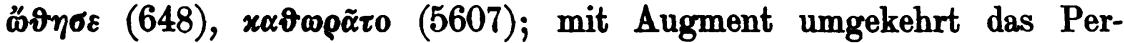

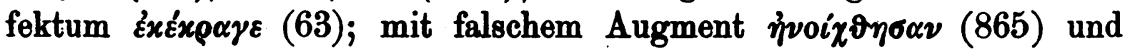
$\pi \rho 0 б \varepsilon \sigma \nu \mu \mu \alpha ́ x \varepsilon \iota$ (591). Ohne Reduplikation bezw. Augment erscheinen:

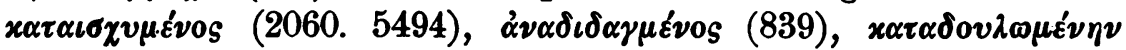

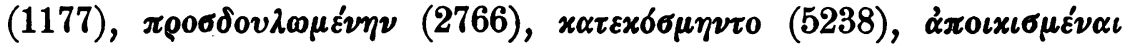

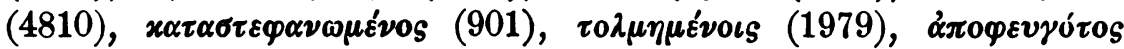

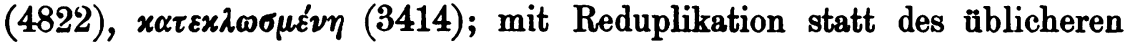

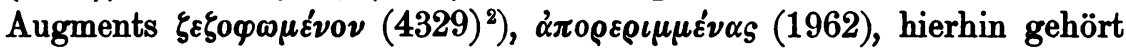

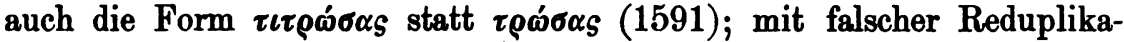

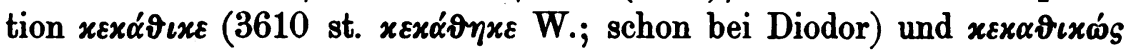
(3519).

Auch in der Tempusbildung finden sich Abweichungen von dem früheren Sprachgebrauch nur in einzelnen Beispielen. Die Verba auf $-\mu \iota$ werden bisweilen als kontrahierende behandelt $\left.{ }^{3}\right)$; so finden sich neben

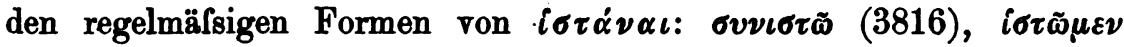

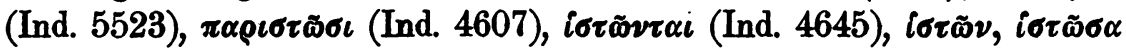

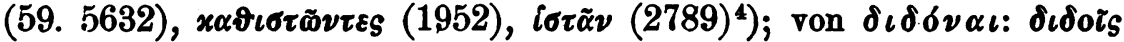

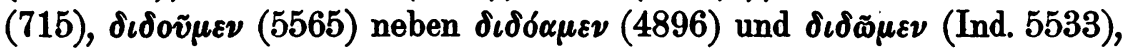

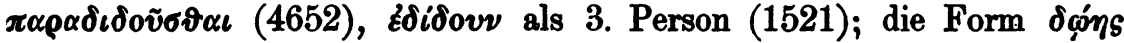
(2301) ist dagegen wohl eine eigene Bildung des Verf., vielleicht nach $\left.\tau \iota \mu \varphi^{\prime}\right)$; von $\tau \iota \vartheta \varepsilon v \alpha \iota$ finden sich abgesehen von $\sigma \dot{v} \nu \vartheta \varepsilon \sigma o \nu$ (1453) nur mit der alten Grammatik übereinstimmende Bildungen. Diese Form, sowie $\delta \omega ́ \sigma \omega ~(927), \delta \omega ́ \sigma \omega \mu \varepsilon v ~(5129.5326)$ als Konjunktiv des Aorists sind die einzigen ungewöhnlichen sigmatischen Aoristbildungen. Von $\varepsilon i \nu \alpha \iota$ finden sich die Formen $\ddot{\eta} \mu \eta \nu$ als Imperfekt (5835), das schon in der Koine nicht ungebräuchlich war $\left.^{5}\right)$, und $\omega_{\mu \varepsilon \nu}$ 'wir sind' (4881. 5041), von der ich nicht anzugeben weifs, ob sie nicht etwa eine besondere Bildung unseres Verf. ist. Eine Analogiebildung scheint dagegen $\pi \rho \delta \sigma \varepsilon \iota \sigma \iota$ 'sie gehen heran' (Präsens st. Aorist, 4002. 4920).

1) Schmid, Att. 1, 228 f. Hatzidakis, Einl. S. 63. 66. 67; vgl. Usener, D. heil. Theod. S. 138.

2) Dieselbe Form in der Bedeutung 'finster' bei Theoph. chron. p. 480, 27 (de Boor).

8) Vgl. Kühner, Gr. Gr. 1, 840. Schmid, Att. I, 229; $2,25 \mathrm{f}$.

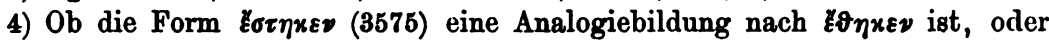
nur ein Schreib- bezw. Druckfehler, wage ich nicht zu entscheiden.

๖) Lobeck, Phryn. p. 152 u. A. Schmid, Att. 1, 231. Winer a. a. 0. S. 76. Kühner, Gr. Gr. 1, 666. Mullach S. 281. 
Der Gebrauch der ersten Aoriste statt der früher üblichen zweiten begegnet von manchen Verben nicht $\operatorname{selten}^{1}$ ); so von $\ddot{\alpha} \gamma \varepsilon \iota \nu$ neben

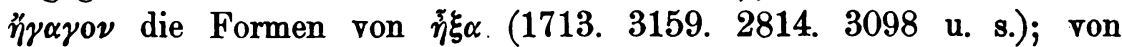

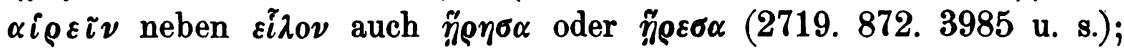
von $\varepsilon \dot{v} \varrho i \sigma x \varepsilon \iota \nu$ auch $\varepsilon \tilde{v} \varrho \eta \sigma \alpha\left(357.2397 .773\right.$ u. s.); von $\lambda \alpha \mu \beta \alpha^{\prime} \nu \varepsilon \iota \nu$ nur einmal $\pi \varrho 0 \sigma \lambda \eta \dot{\psi} \psi \iota \mu \iota$ (1054); von $\lambda \varepsilon i \pi \varepsilon \iota \nu \varepsilon \tilde{\varepsilon} \lambda \varepsilon \iota \psi \alpha$ (2129. 3949. 142 u. s.); von $\tau i x \dot{\tau} \varepsilon \iota \nu$ nur einmal $\tau \dot{k} \xi \alpha \iota$ als Infinitiv (227); von

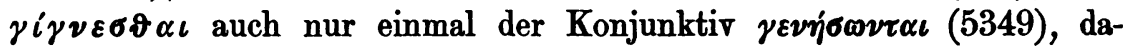
gegen erscheint der in der späteren Gräzität nicht seltene Aorist $\dot{\varepsilon} \gamma \varepsilon \nu \eta^{\prime} \vartheta \eta \nu$ statt $\dot{\gamma} \gamma \varepsilon \nu{ }^{\mu} \mu \eta \nu$ auch bei unserm Verf. mehrfach (2754. 3904 u. s.). $\mathrm{Zu}$ den Abweichungen in der Form gehört es auch, wenn der Verf. von manchen Verben einen medialen Aorist statt des aktiven ge-

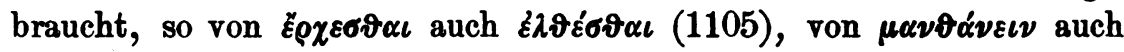
$\mu \alpha \vartheta \varepsilon \sigma \vartheta \alpha \iota$ (1673. 3433 u. s.) nur im Infinitiv, von $\delta \rho \tilde{\nu} \nu-\imath \xi \varepsilon \sigma \theta \alpha \iota(314)$; $\mu \alpha^{\prime} \chi \varepsilon \sigma \vartheta \alpha \iota$ bietet folgende Formen: $\mu \alpha \chi \eta^{\prime} \sigma o \mu \alpha \iota$ (2194. 2759), ź $\mu \alpha \chi \varepsilon \sigma \alpha \dot{\alpha} \mu \eta \nu$

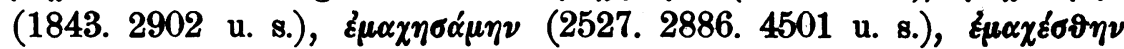

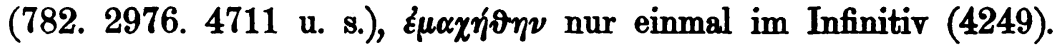

Aufserdem zeigt sich bei unserm Verf. Verwirrung in den kontrahierenden Verben, insofern einmal einfache Verba auf $-\omega$ als solche

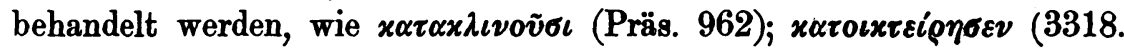

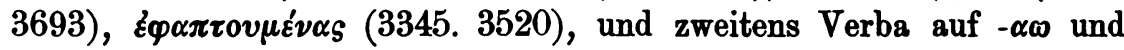
$-\varepsilon \omega$ in einander übergehen $\left.{ }^{2}\right)$; so begegnen von $\alpha \nu \tau \tilde{\alpha} \nu-i \pi \tau v \tau$ -

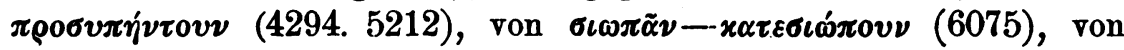

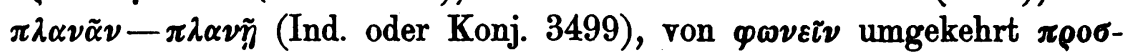

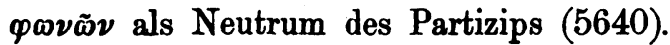

Schliefslich führe ich als Einzelheiten, deren Bildung unser Verf. sich selbst erlaubt hat, an $\gamma^{\prime} \nu \omega \mu \varepsilon \nu$ von $\gamma^{\prime}(\gamma \nu \varepsilon \sigma \vartheta \alpha \iota$ (4802) und $x \alpha \tau \alpha-$

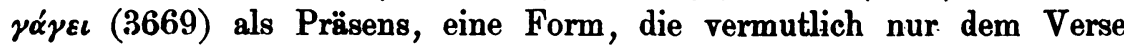
ihre Entstehung verdankt.

\section{Adverbia.}

Was die Adverbia anbelangt, so ist von $\mathrm{Krumbacher}^{3}$ ) darauf aufmerksam gemacht worden, dals das vulgärgriechische $\alpha x \mu \eta \nu$ in der Bedeutung 'noch' sich viermal in unserm Gedichte findet, während sonst

1) Lobeck, Phryn. p. 28 A. Hatzidakis, Einl. S. 176. Sollte vielleicht auch

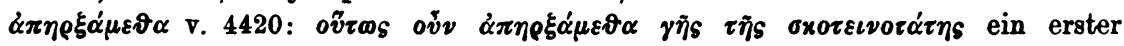

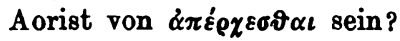

2) Vgl. Hatzidakis, Z. Präsensbildg. des Neugriech., Kuhns Ztschr. 27, 71; Neugr. Miscellen, Ztschr. 33, 106 f., und Einl. S. $128 \mathrm{f}$.

3) In Kuhns Ztschr. 29, 189, nämlich v. 1103. 1705. 2323. 3546. Über \& $x \mu \eta \dot{\eta} v$ und dessen Entstehung und Bedeutung vgl. dens., ebenda 27, $498 \mathrm{ff}$. 
Ė von dem Verf. angewandt wird. Sonst schliefst sich der Verf. durchaus an die alte Sprache an; ich bemerke nur, dafs die Adverbialbildung auf $-\alpha$, die schon bei den früheren Byzantinern auftritt, nur in $\tau \alpha \dot{\alpha} \chi \alpha$ erscheint, das sehr oft vorkommt, während $\tau \alpha \chi \varepsilon ́ \omega s$ nur dreimal (169. 2764. 4992) begegnet. Aufserdem weise ich darauf hin, dafs die Adverbia auch im Superlativ oft auf $-\omega_{s}$ neben dem Neutr. Pl. auf $-\alpha$ gebildet werden, $\tau \alpha \chi i \sigma \tau \omega s$ (22mal), $\tau \alpha \chi v \tau \alpha^{\prime} \tau \omega s$ (5mal), $\tau \alpha^{\prime} \chi\llcorner\sigma \tau \alpha$ dagegen $41 \mathrm{mal}$ und $\tau \alpha \chi v ́ \tau \alpha \tau \alpha$ sehr oft; von $x \alpha \lambda \tilde{\omega} s$ heifst der Super-

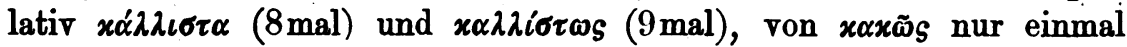
xóx

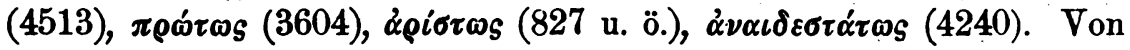
Partizipien gebildete Adverbia ${ }^{1}$ ) begegnen in $\lambda \alpha \nu \vartheta \alpha \nu \sigma \delta \tau \omega s$ (1953),

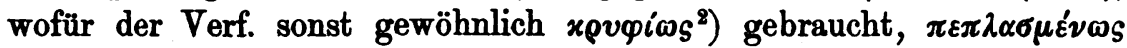

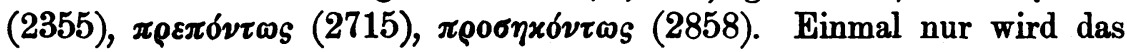
Neutr. Sing. adverbial gebraucht (2058), und zweimal sogar das Maskulinum falsch in adverbialem Sinne gesetzt (äxv́s 3519 und $\pi \rho \tilde{\tau}$ os

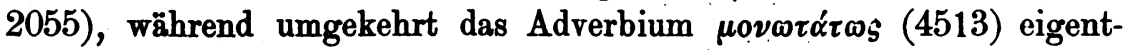
lich Adjektiv sein mülste.

In betreff der Negationen genügt die Bemerkung, dafs unser Verf. ov und $\mu \dot{r}$ völlig gleichbedeutend anwendet.

1) Vgl. Schmid, Att. 2, 54; $3,77$.

2) Dies Adverbium ist vermutlich auch v. 3605 statt xovqioıs herzustellen:

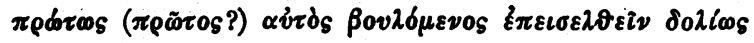

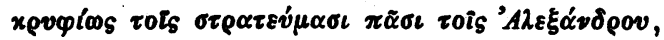

d. h. Darius will listig und heimlich Alexanders Heer überfallen. Die Auslassung des 'und' kann bei unserm Verf. nicht auffallen; denn er läst es vielfach aus zwischen zwei Begriffen, wo es eigentlich stehen mürste. Ich begnüge mich einige

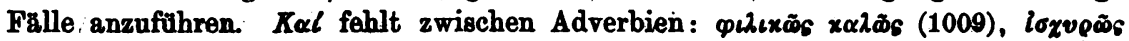

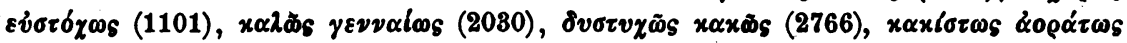

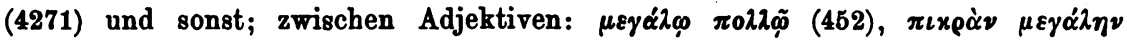

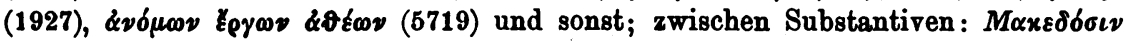

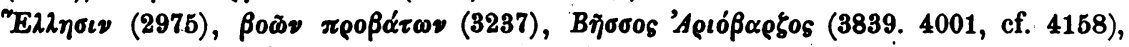

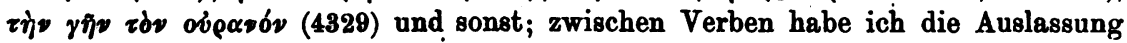

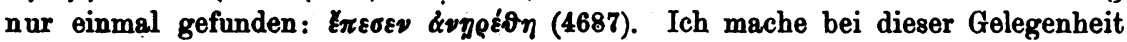
zugleich darauf aufmerksam, dafs auch die unmotivierte Einschiebung von $x \alpha l$

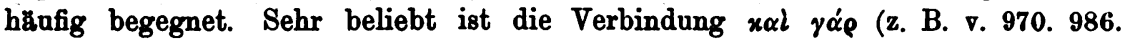
1766. 1844. 2038. 2477 u. s. w.) und $x \alpha l$ ród $\alpha_{\iota \nu}$ (296. 385. 1035. 1369. 2495.2569 u. s. w.), aufserdem aber auch bei Adjektiven, besonders bei róvos (z. B. 490. 4204. 6512 u. 8. w.), bei Partizipien in Verbindung mit einem Relativum (ôs $x \alpha l$ $\mu \alpha \vartheta \dot{\omega} \nu$ 1285, ô $\nu \alpha \alpha$ $\lambda \alpha \beta \dot{\omega} \nu$ 1579, ferner 1739. 2996. 3464. 4230. 4521. 5616. 5967) und Pronominibus (3224. 3771. 4742). 


\section{Syntax.}

\section{Subjekt und Prädikat.}

Die Beziehungen von Subjekt und Prädikat entsprechen durchaus den Forderungen der alten Grammatik; denn dals zu dem Subjekt im Neutr. Pl. auch das Prädikat im Plural tritt, ist eine auch in der klassischen Sprache so häufige Erscheinung, dafs ich füglich von einer Anführung von Beispielen Abstand nehmen kann. Bemerken dagegen möchte ich, dafs die schon aus Homer bekannte umgekehrte Assimilation des Subjekts bei unserm Verf. sich einmal findet v. 395: $\tau \grave{o} v$

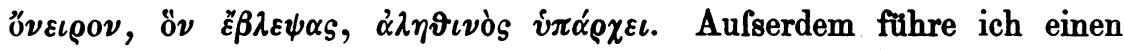
für die grammatische Klarheit unseres Verf. recht bezeichnenden Satz an, in welchem das Prädikat auf das Objekt eines zu dem Subjekt dieses Satzes gehörigen Partizips bezogen ist, nämlich v. $5820 \mathrm{ff.:}$

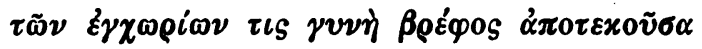

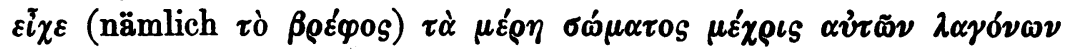

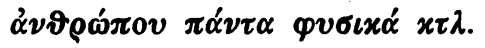

Es ist hier die Rede von der Mifsgeburt, durch welche Alexanders Tod vorausverkündigt wird; für jeden, der die Sache nicht anderweitig kennt, mufs nach dieser Stelle eigentlich die Frau selbst diese Mifsgeburt sein.

\section{Attribut und Apposition.}

Die Beziehungen dieser Satzteile zeigen einige Eigentümlichkeiten in Genus und Kasus, die auf den Einflufs der Volkssprache hinweisen. Zunächst stimmt an manchen Stellen das Genus nicht überein, vielleicht weil die Formen in gewissem Sinne schon erstarrt waren ${ }^{1}$ ), so: $\tau i$

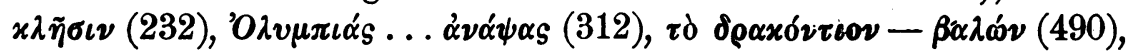

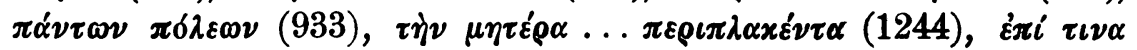

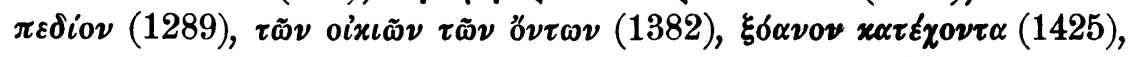

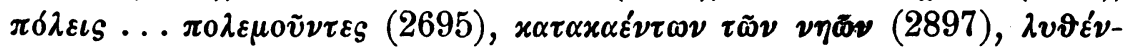

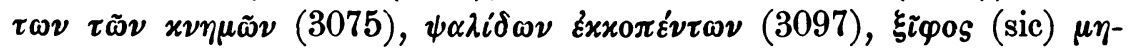

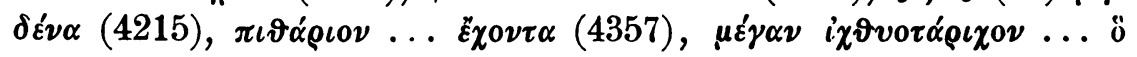

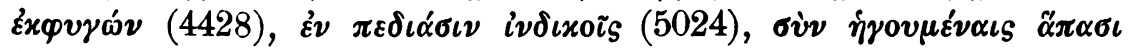
(5492), हैं nach 5376). ${ }^{2}$ )

1) S. Krumbacher in Kuhns Ztschr. 29, 190, wo einige der obigen Beispiele schon angeführt sind. Eine so erstarrte Form scheint bei unserm Verf. auch

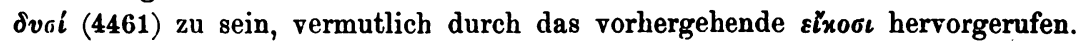

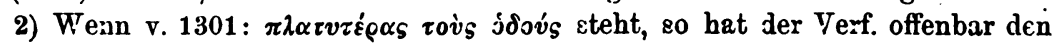
Artikel der Endung des Substantivs assimiliert, wenn nicht ein Fehler des Abschreibers vorliegt. 
Ferner-kommt es nicht ganz selten vor, dafs die Apposition in einem andern Kasus ${ }^{1}$ ) steht als das zu erklärende Substantiv; teilweise, bei längeren Sätzen, war dem Verf. offenbar die Beziehung verloren gegangen, teilweise war der Sinn für die Notwendigkeit derselben wohl

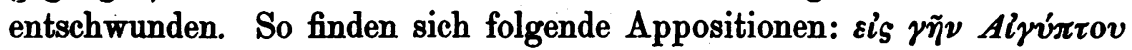

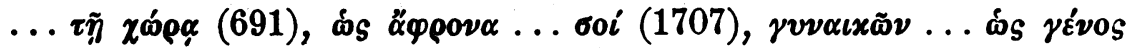

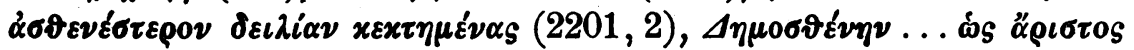

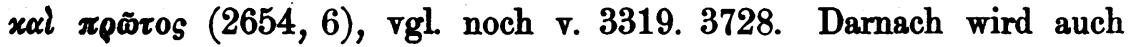
v. $2276 \mathrm{ff}$ :

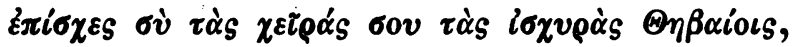

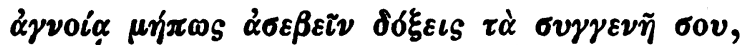

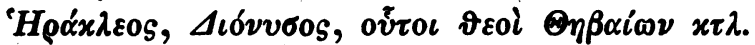

nach бov Komma (statt des Punktes bei Wagner) zu setzen und der

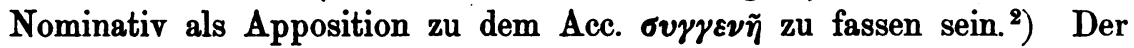
Sänger Ismenias will Alexander eben erklären, dafs er Thebaner ist und verwandt mit den thebanischen Göttern und Heroen.

\section{Kasus.}

In der Rektion der Kasus zeigt sich bei unserm Verf. am meisten der Einflufs der Volkssprache, insofern der Accusativ, der ja schon seit Diodor $^{3}$ ) eine allmählich immer mehr herrschende Stellung einnimmt, auch bei $\mathrm{ihm}$ eine bedeutende Ausdehnung gewonnen hat. Freilich sucht er dann andrerseits wohl seine Gelehrsamkeit dadurch zu erweisen, dafs er auch die andern Kasus, diese dann allerdings oft ganz ungehörig, zur Anwendung bringt.

Was zunächst den Accusativ anbelangt, so werden die Verba des

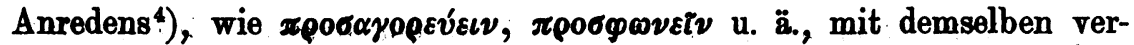

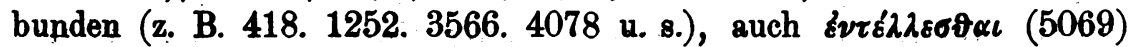

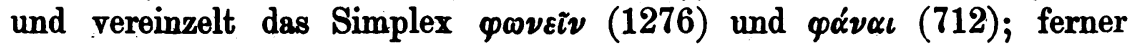
$\dot{\alpha} x 0 \lambda 0 v \vartheta \varepsilon i \nu, x \alpha \tau \alpha x 0 \lambda o v \vartheta \varepsilon i \nu$ (3386. 3078), $\alpha \nu \tau \alpha \tilde{\nu} \nu$ mit seinen Kompositis (2235. 5341. 5483 u. s., $\pi \rho 0 \sigma \alpha \pi \alpha \nu \tau \tilde{\alpha} \nu$ und $\pi \rho \circ \sigma v \pi \alpha \nu \tau \tilde{\alpha} \nu$ mit Dativ

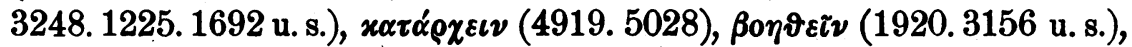

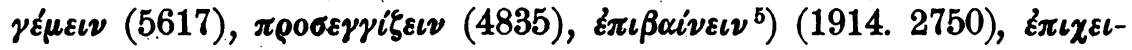

1) Das Attribut stimmt im Kasus immer überein; denn v. 4700 ist für $\pi$ iqelovs

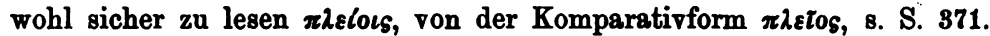

2) Als Parallele zu dieser Beziehung des Nominativs auf den Accusativ diene

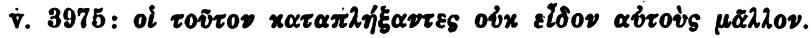
S. $220 \mathrm{f}$.

3) Vgl. F. Krebs, Z. Rektion der Kasus, Heft 1 \& ; Hatzidakis, Einl.

4) Krebs a. a. O. Heft 3 S. 9.

5) Vgl. Kühner, Gr. Gr. 2, 299 A. 7; Krebs; Progr. Regensbg. 1885 S. 13, und Z. Rektion der Kasus, Heft 2 S. 17. 


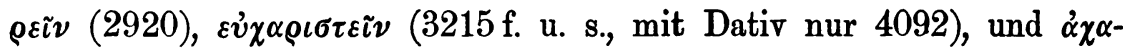

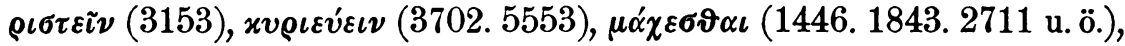

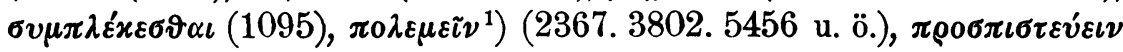

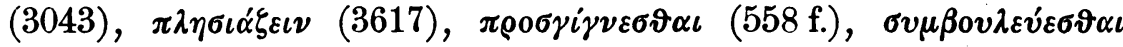

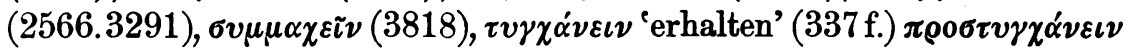

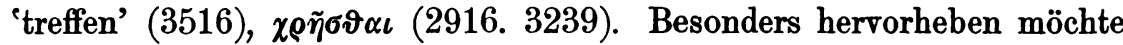

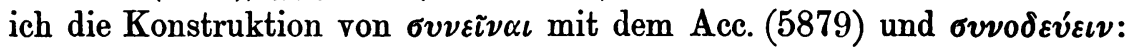

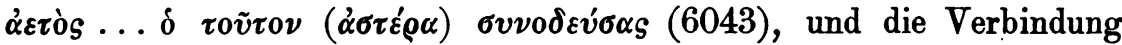

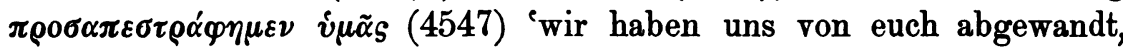
euch links liegen lassen'. Interessant ist besonders auch die Konstruktion des doppelten Accusativs, die unser Verf. mit entschiedener Vorliebe bei einer ganzen Reihe von Verben benutzt; sie findet sich häufig z. B. bei $\left.\delta \iota \delta \delta \nu \alpha \iota{ }^{2}\right)$ und sinnverwandten Verben, so: $\tau \iota \mu \eta \dot{\nu} x \alpha \imath$

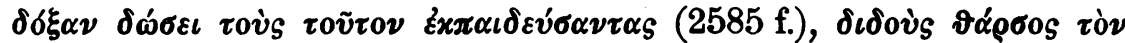
$\alpha v ่ \tau \delta$ (2943), fermer 3025. 5256, auch in der Bedeutung 'treffen': dòs

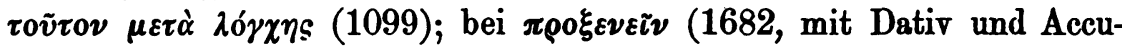

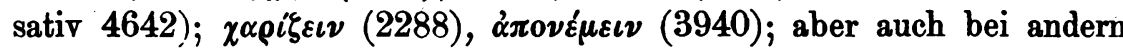

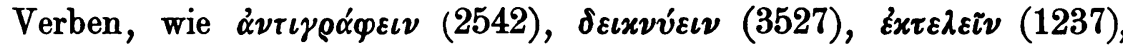

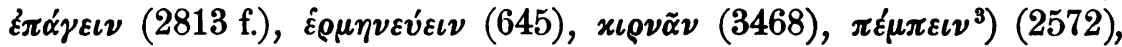

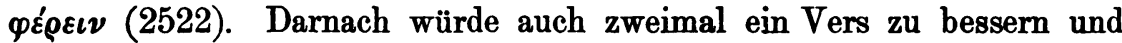
zu erklären sein, der in der Wagnerschen Rezension nicht richtig sein kann. Als die Brahmanen von Alexander hören, dafs auch er sterblich ist, rufen sie u. a. aus:

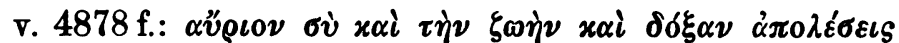

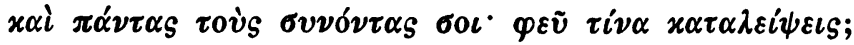

So können diese Verse nicht in Ordnung sein, denn die Frage: „wen wirst du zurücklassen?" ist in diesem Zusammenhange eigentlich sinnlos; es wird nur mit Änderung der Interpunktion zu lesen sein: $x \alpha i$

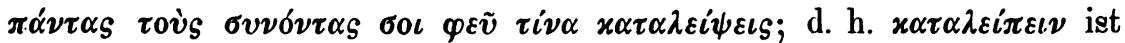
mit dem doppelten Acc. verbunden, und ebenso ist es v. 6018: $\dot{\eta} \mu \tilde{\alpha} s$

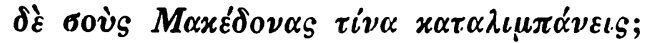

1) Vgl. Krebs a. a. O. Heft 1 S. 5 ff.

2) Vgl. Ind. Graec. z. Theophan. bei de Boor s. v. $\delta \omega \varrho \varepsilon i \sigma \vartheta \alpha \iota$.

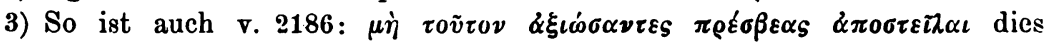
Verbum mit dem doppelten Acc. zu konstruieren. Etwas zweifelhaft ist eine Stelle, wo es von Alexandria heifst v. 2780 f.:

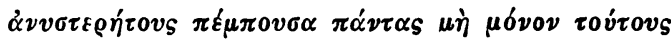

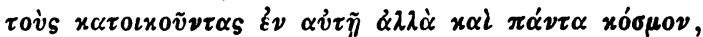

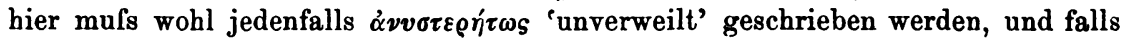

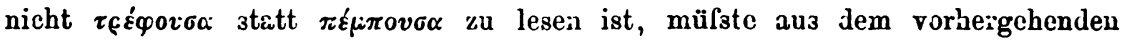
Verbum $\vartheta \rho \dot{\psi} \psi \varepsilon \iota \varepsilon$ ein Substantiv wie $\tau \rho \circ \varphi \eta \dot{\nu}$ zu $\pi \dot{\mu} \mu \pi \sigma v \sigma \alpha$ ergänzt werden, sodals auch hier der doppelte Acc. stände.

Bguant. Zeitsohrift VII 2. 
Ist so der Accusativ seiner Herrschsucht entsprechend der eigentlich dominierende Kasus geworden, so hat doch auch der Dativ sich, wie schon bemerkt wurde, bei unserm Verf. einige Gebiete erobert ${ }^{1}$ ), wohin er eigentlich nicht gehört. Ich sehe dabei ab davon, dafs bei den Verben gehen, kommen, werfen, fallen u. $\ddot{a}$. der Dativ oft die Richtung auf die Frage wohin? ausdrückt ${ }^{2}$ ), z. B. $x \alpha \tau \varepsilon \lambda \vartheta \varepsilon \tilde{\nu} \tau \tilde{\omega} \boldsymbol{\varphi} \beta \dot{v} \vartheta \omega$

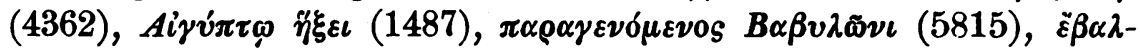

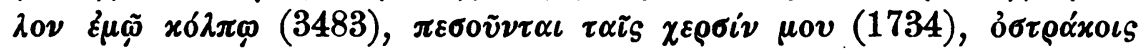

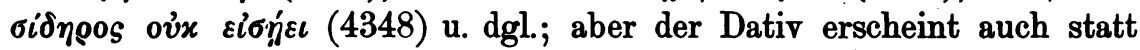

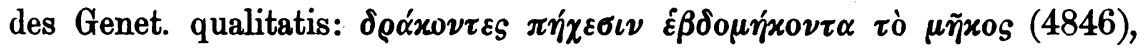
ferner statt des sonst üblichen Acc. des Bezuges $\tau \tilde{\omega} \mu \tilde{\eta} x \varepsilon \iota$ (4207), $\tau \tilde{\omega}$ $\sigma \tau \alpha \vartheta \mu \tilde{\omega}$ (4320), ferner, um die Ausdehnung zu bezeichnen, lokal: $x \alpha \tau-$

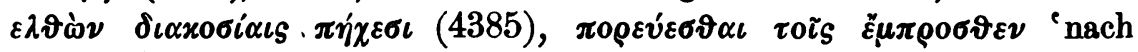

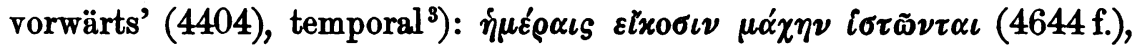

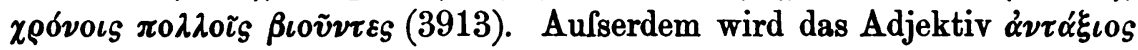
mit dem Dativ verbunden (4922) und Verba, die

1) sonst transitiv gebraucht werden, nämlich:

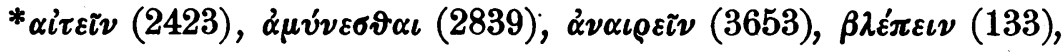

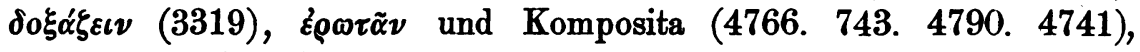
$x \alpha \vartheta \iota x \varepsilon \tau \varepsilon v \varepsilon \varepsilon v(4516), x \alpha \tau \alpha \delta v \sigma \omega \pi \varepsilon i \nu(1500), *_{x \alpha \tau \alpha \lambda \alpha \mu \beta \alpha^{\prime} \nu \varepsilon \iota \nu}(5544), *_{x \alpha-}$

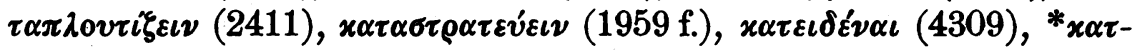
$\varepsilon \mu \pi 0 \delta i \xi \varepsilon \iota \nu$ (3294), $x \varepsilon \lambda \varepsilon v \varepsilon \varepsilon \nu$ (757 f. 1340 u. ö.), $\lambda \alpha \nu \vartheta \alpha^{\prime} \nu \varepsilon \iota \nu$ (1951), $\lambda \varepsilon i \pi \varepsilon \iota \nu$

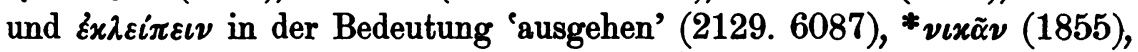

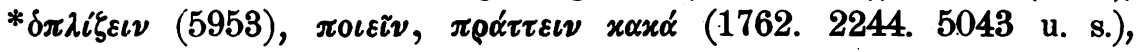

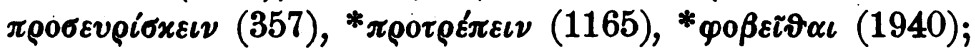

2) sonst gewöhnlich mit dem Genetiv verbunden werden, wie:

$\dot{\alpha} \xi \iota 0 \tilde{\nu}$ (1056), $\varepsilon \gamma \gamma i \xi \varepsilon \iota \nu$ (751), $\dot{\varepsilon} \pi \iota \tau v \gamma \chi \alpha \dot{\alpha} \nu \varepsilon \nu$ 'erhalten' (3053),

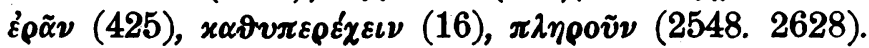

Endlich mache ich darauf aufmerksam, dafs, wie es scheint ${ }^{4}$ ), auch ein absoluter Dativ $^{5}$ ) bei unserm Verf. begegnet:

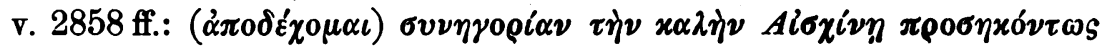

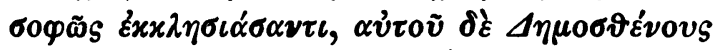

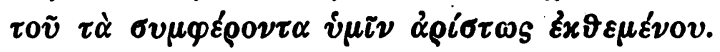

1) Auf diese Eigentümlichkeit unseres Verf. macht Krumbacher in Kuhns Ztschr. 29, 190 aufmerksam; ich bezeichne die von ihm schon angeführten Verba mit einem Stern.

2) Vgl. Kühner, Gr. Gr. 2, $351 \mathrm{f}$.

3) Vgl. Schmid, Att. 1. 92, 236; 2, $41 \mathrm{f}$; 3, $55 \mathrm{f}$.

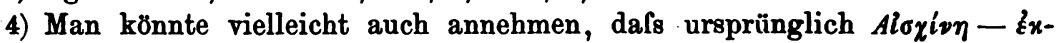
$x \lambda \eta \sigma \iota \alpha^{\prime} \sigma \alpha \nu \tau o s$ gestanden hätte und später geändert wäre; indessen ist ja der absolute Dativ durchaus möglich.

5) S. Schmid, Att. 1, 230; $2,42$. 
Zum Schlufs ist endlich hervorzuheben, dafs auch der Genetiv bei unserm Verf. wieder etwas zu Ehren gekommen ist, gewöhnlich freilich, wo er wenig angebracht war. So hat er vermutlich eine dunkle Ahnung, dafs $x \alpha \tau \alpha$ und $\pi \varepsilon \rho i$ mit dem Genetiv verbunden werden; daher konstruiert er denn $\varkappa \alpha \tau \alpha \sigma \pi \alpha^{\prime} \xi \varepsilon \sigma \vartheta \alpha \iota$ (1003), $x \alpha \tau \alpha \mu \alpha \nu \tau \varepsilon v \varepsilon \varepsilon \sigma \vartheta \alpha \iota$ (nicht etwa in ungünstigem Sinne, 2175) und darnach auch das einfache $\mu \alpha v$ -

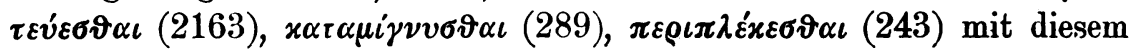

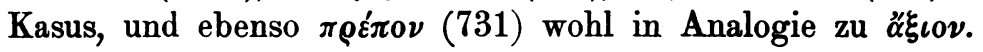

\section{Präpositionen. ${ }^{1}$ )}

In der Rektion der Präpositionen ist von Abweichungen in betreff des Kasus nur zu bemerken, dafs ámó, wie ja schon in den ersten Jahrhunderten unserer Zeitrechnung, an fünf Stellen mit dem Accusativ verbunảen ist (1343. 3477. 3942. 5748. ${ }^{2}$ ) 6093). Zweifelhaft dagegen erscheint es, ob $\varepsilon i s$, das sonst stets den Accusativ bei sich hat, an einer Stelle mit dem Dativ zu konstruieren ist. Es heifst v. 4824:

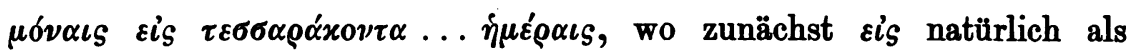
Präposition gefalst wird. Da indessen der Dativ auf die Frage wie lange? bei unserm Verf. durchaus gebräuchlich ist und ferner Präpo-

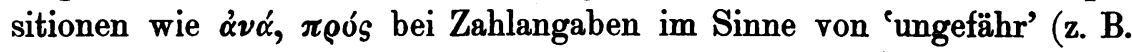

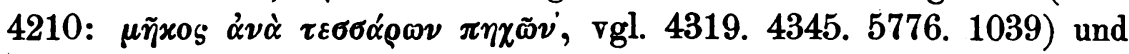

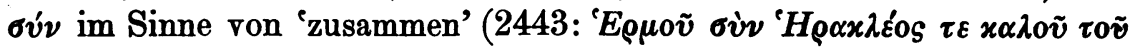
$\Delta \iota$ vข์бov) durchaus adverbial gebraucht werden, so wird auch $\varepsilon i s$ hier in dem Sinne von 'ungefähr' zu fassen und die Verbindung mit dem Dativ nicht anzunehmen sein.

Über die Anwendung der Präpositionen ist Folgendes zu bemerken. Der Gebrauch von $\varepsilon^{\prime} \nu$ statt $\left.\varepsilon i \varsigma^{3}\right)$ ist so gewöhnlich (33mal), dafs ich

1) Die Form der Präpositionen ist die bei den klassischen Schriftstellern gebräuchliche; nur einmal würde der Verf., wenn meine Vermutung richtig ist, die vulgär- und neugriechische Form $x \alpha^{\prime}$ für $x \alpha \tau \alpha ́$ (s. Hatzidakis, Einl. S. 152) gebrauchen, v. $\mathbf{4 4 5 9}$ f.:

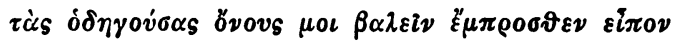
$x \alpha i \tau \grave{\eta} \nu \tau \tilde{\omega} \nu \not{\alpha} \sigma \tau \rho \omega \nu \ddot{\alpha} \mu \alpha \xi \alpha \nu$.

Alle andern Bearbeitungen haben, wenn sie auch sonst von einander abweichen,

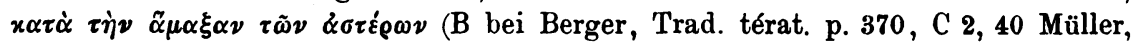
L p. 766 Meusel), und $x \alpha i$ giebt hier in der That auch keinen rechten Sinn. Durch die Änderung des $x \alpha i$ in $x \alpha \dot{\alpha}$ kommt alles in Ordnung.

2) Hier liest Kapp, Mitteilungen aus zwei griech. Hss, Pr. d. Obergymn.

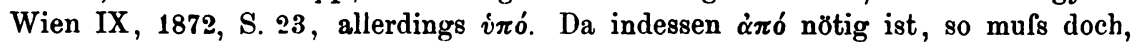
falls auch in der $\mathrm{Hs}$ i $\pi c^{\prime}$ steht, gebessert werden.

3) Vgl. im allgemeinen Krumbacher in Kuhns 'Ztschr. 27, $543 \mathrm{f}$. Einige Beispiele aus unserm Verf. führt derselbe, ebenda 29, 191, an; vgl. Usener, D. heil. Theod. S. 129. 
die Aufzählung der Stellen füglich unterlassen kann (man vgl. z. B.

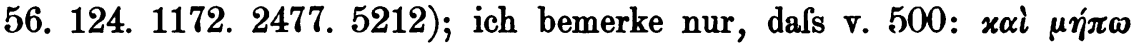

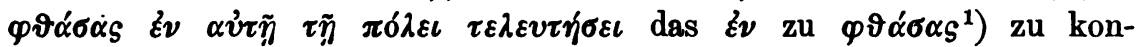
struieren ist. Dals $\varepsilon i_{S}$ statt $\dot{\varepsilon} v$ gesetzt wird, ist zwar nicht so häufig,

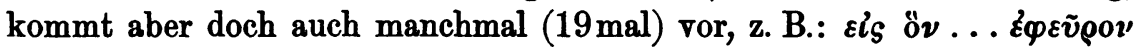
(181, ebenso an der entsprechenden Stelle des Ps.-Kall. 1, 4), $\mu \varepsilon \dot{v \varepsilon \varepsilon \nu}$

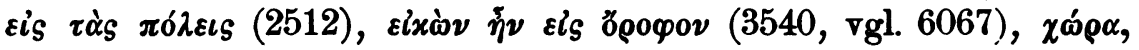

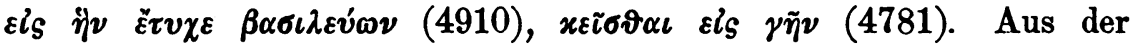
theologischen Bildung des Verf. stammt wohl der Gebrauch von $\varepsilon i s$

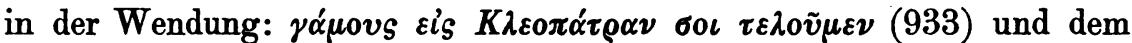
entsprechend $\varepsilon l_{S} \stackrel{\alpha}{\alpha} \nu \rho \alpha \alpha \alpha \mu \beta \alpha \dot{\nu} \nu \varepsilon \iota \nu$ (4091, vgl. Sept. Genes. 34, 12). Auch die, Anwendung von $\varepsilon l_{S}^{2}$ ) für $\pi \rho o ́ s, \pi \alpha \rho \alpha ́$, ம́s mit dem Acc., ja selbst von $\dot{\varepsilon} \nu$ ( nicht durchaus ungewöhnlich.

In derselben Weise bezeichnet nun bei Präpositionen, die den Dativ oder Accusativ regieren, der erstere die Bewegung, der zweite die Ruhe, oder es werden beide Kasus in derselben Bedeutung gesetzt. So wird z. B. Tœós fast ganz gleichbedeutend gebraucht mit 'in, bei',

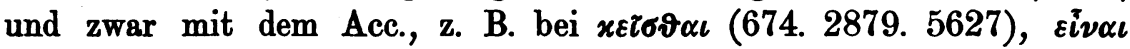

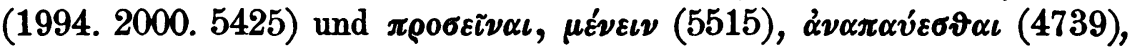

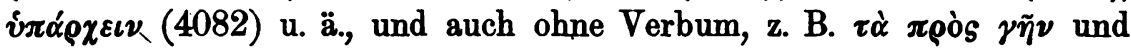

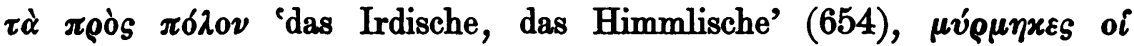

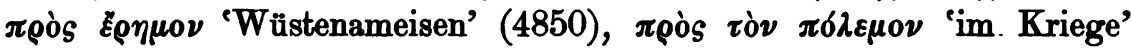
(5530) u. s. w. Umgekehrt tritt dann wieder der Dativ ein zur

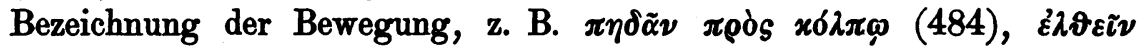

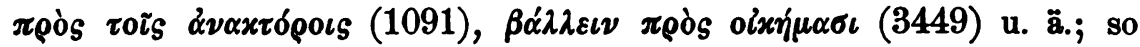
wird dann auch $\pi \rho \delta ́ s$ mit dem Dativ gleichbedeutend mit $\varepsilon_{\nu}$ gebraucht,

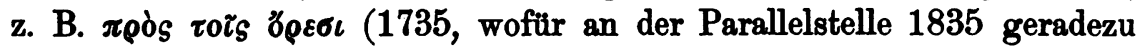

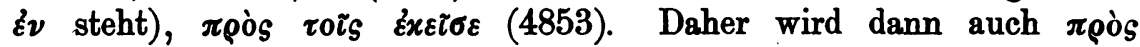

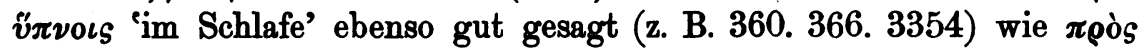
ช̇uvovs (3383), und endlich wird auch in demselben Satze der Dativ

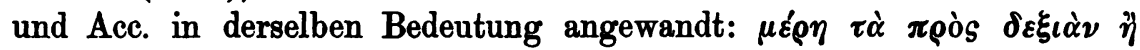

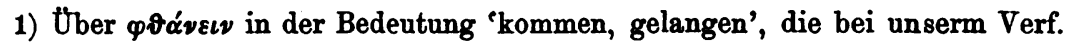
ganz gewőhnlich ist, vgl. Usener, D. heil. Theod. S. 131. 175. Unser Verf. gebraucht das Verbum auch in der Bedeutung 'zu etwas kommen': ovx ž

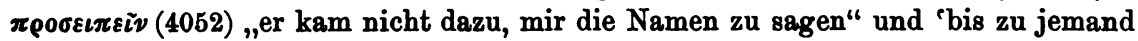

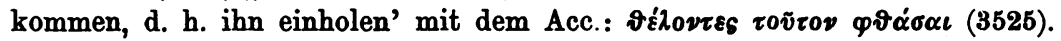

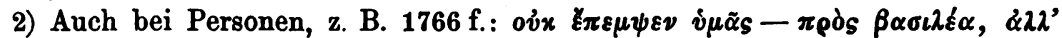

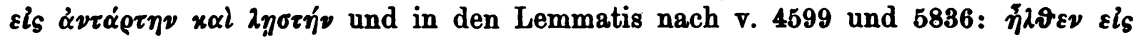
$\tau \dot{\nu} \nu$ 'A $A \xi \xi \alpha \nu \delta \rho o v$. Vgl. über diesen Gebrauch Krebs, Die Präpositionsadverbien 2. Teil. S, 62 f. 


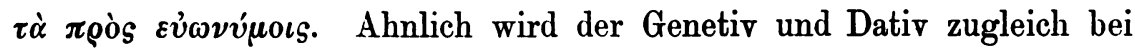

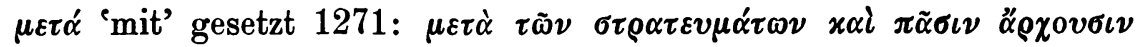

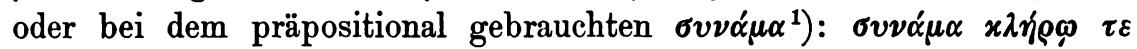

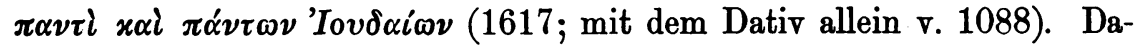
gegen wird $\tilde{\alpha}^{\prime \prime} \mu \alpha$ wieder unbedenklich mit dem Acc. verbunden: $\tilde{\alpha} \mu \alpha$

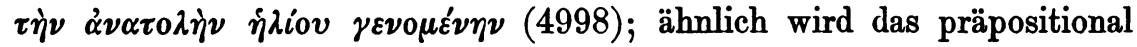
gebrauchte $\mu \varepsilon ́ \sigma o \nu$ zwar gewöhnlich mit dem Genetiv (1696. 2143. 4947 u. s.), aber auch mit dem Dativ verbunden (1805). Ja auch бv́v

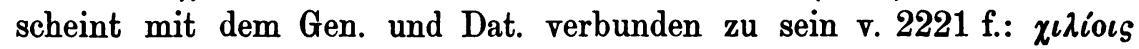
$\sigma \dot{v} \nu \dot{\alpha} \nu \delta \rho \alpha ́ \sigma \iota ~ \gamma \varepsilon \nu \nu \alpha i \omega \nu ~ \tau \varepsilon \sigma \varphi \varepsilon \nu \delta o \nu \eta \tau \tau \tilde{\omega} \nu$. Wie mit $\pi \rho o ́ s$ verhält es sich

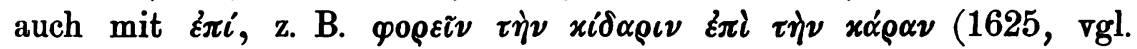
3357) oder $\mu \dot{\varepsilon} \nu \varepsilon \iota \nu$ żंi $\delta \varepsilon \tilde{\pi} \pi \nu 0 \nu$ (5971), umgekehrt steht der Dativ bei

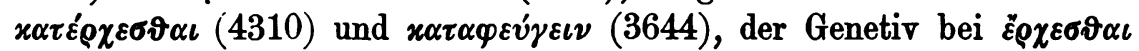

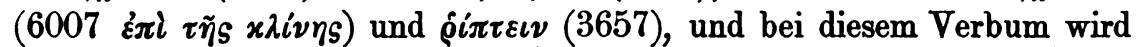
auch $x \alpha \tau \alpha^{\prime}$ c. gen. in derselben Bedeutung verwandt (895). Endlich

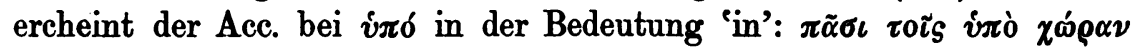

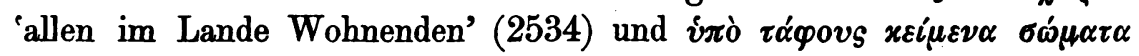
(3637, vgl. 4075).

Unter den Eigentümlichkeiten ist ferner anzuführen, dafs beim

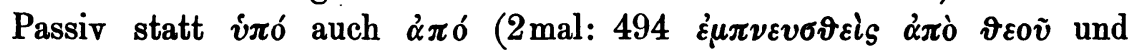

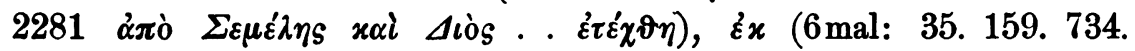
1034. 1423. 4984, vgl. 4975) und ganz besonders $\pi \alpha \rho \alpha^{2}$ ) (nicht weniger als $73 \mathrm{mal}$ ) steht; und zwar steht dies nicht nur bei Personen, sondern auch z. B. bei $\lambda \iota \mu o ́ s$ fürr den einfachen instrumentalen Dativ ( $\alpha \dot{\pi} \alpha^{\prime} \lambda \lambda v \sigma \vartheta \alpha \iota$

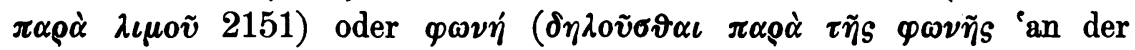

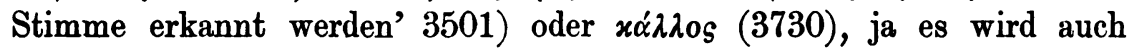

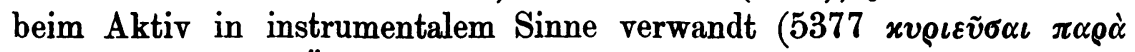

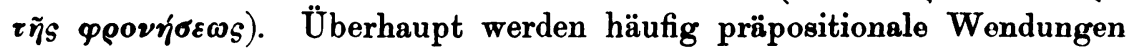
gebraucht, wo der einfache Kasus genügen würde. So finden sich in ähnlicher Weise wie $\pi \alpha \rho \alpha ́$ instrumental gebraucht: $\delta \iota \alpha^{\prime}$, z. B. 405,3713

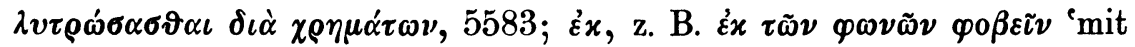

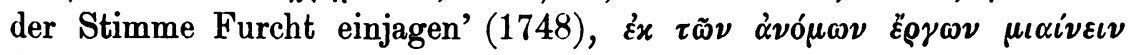

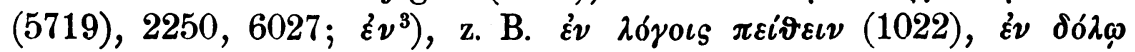

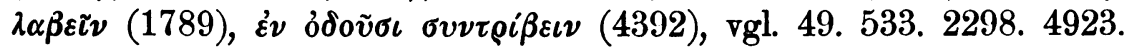

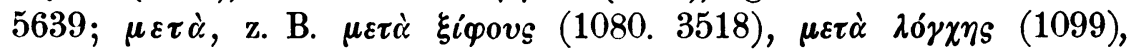
5526. 5342. 5379, $\mu \varepsilon \tau \dot{\alpha} \pi \lambda 0 \iota \alpha \rho i \omega \nu$ 'zu Schiff' 4334, $\mu \varepsilon \vartheta '$ ï $\pi \pi \omega \nu$ 'zu

1) Vgl. dazu Krebs a. a. O. S. 44 ff.; Hatzidakis, Einl. S. 214; de Boor, Ind. Graec. zu Theoph. chron. s. v. $\tilde{\alpha} \mu \alpha$.

2) Vgl. Hatzidakis, Einl. S. 211. Einmal steht sogar $\pi \alpha \rho \alpha$ c. dat. bei

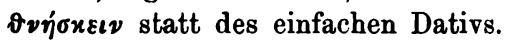

3) Vgl. Usener, D. heil. Theodos. S. 124. 


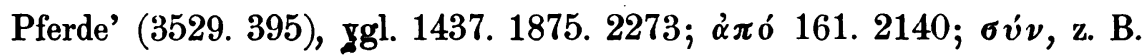

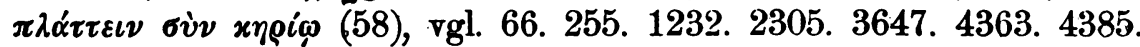
Ferner wird zum partitiven Genetiv ${ }^{1}$ ) nicht nur sehr häufig हैx (z. B.

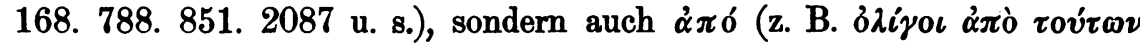

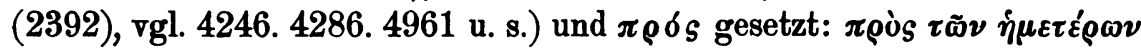

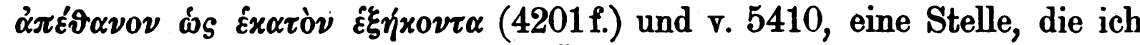
ganz hersetze, weil eine kleine Änderung in der Interpunktion des Textes bei Wagner vorgenommen werden mufs. Es ist die Rede von der Götterhöhle:

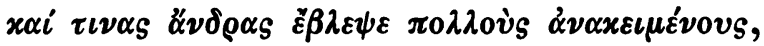

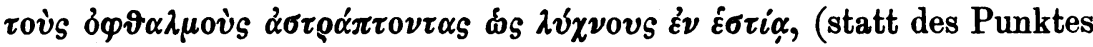
bei W.)

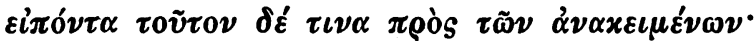

Der letzte Vers ist zu übersetzen: „und er (Alexander) hörte (leicht aus $\check{\varepsilon} \beta \lambda \varepsilon \psi \varepsilon$ zu entnehmen), wie einer der Liegenden zu ihm (d. h. Alexander;

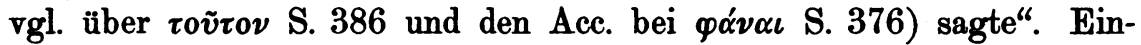
mal scheint sogar $\pi \alpha \rho \alpha^{\prime}$ mit dem Gen. in diesem Sinne zu stehen: $\dot{\omega} S$

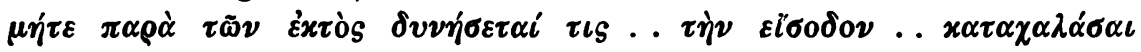
(5782). In einem Falle steht für den Genet. bei Zahlangaben ảró (4298); dieselbe Präposition wird auch zur Angabe des Stoffes ver-

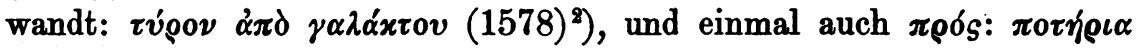

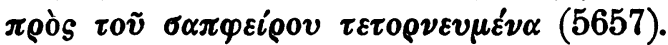

Präpositionale Verbindungen statt eines einfachen Kasus begegnen

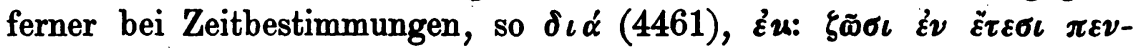

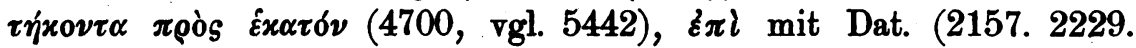
3737) und Acc. (3064). Sehr beliebt ist die Bezeichnung der Art und Weise oder des begleitenden Umstandes durch $\mu \varepsilon \tau \alpha ́$ und zwar auch mit einem Attribut, wobei als Eigentumlichkeit hervortritt die prădikative Stellung des Adjektivs rodv́s im Positiv und Superlativ; andere Attri-

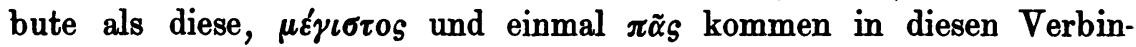

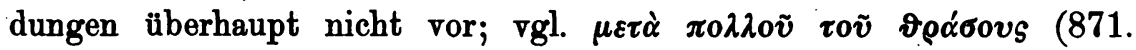

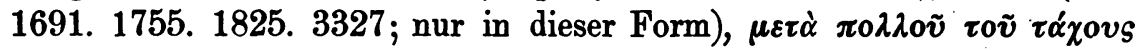

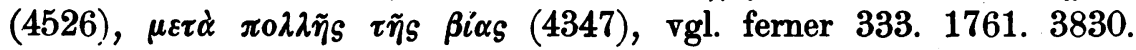

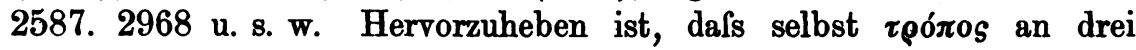

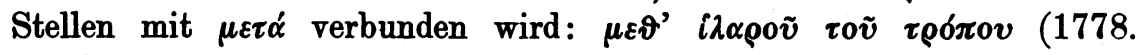

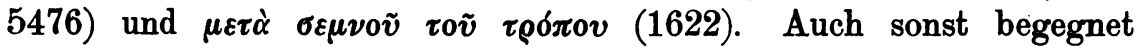

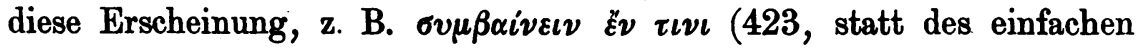

1) Vgl. Hatzidakis, Einl. S 212. Kühner, Gr. Gr. 2, 291 A. 4.

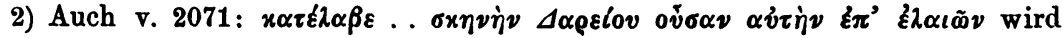
statt $\varepsilon \pi^{\prime}$ zu lesen sein $\alpha \pi^{\prime}=$ aus Olivenholz. 


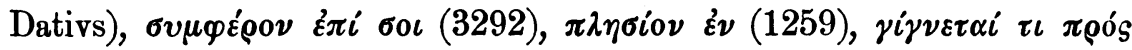

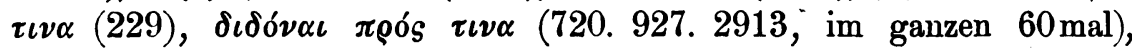

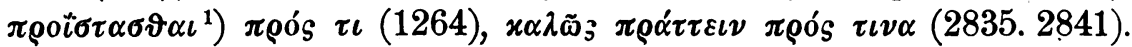
Eine andere Präposition als die früher gebräuchliche oder der Dativ tritt ein bei $\mu \alpha^{\prime} \chi \varepsilon \sigma \vartheta \alpha \iota$ und sinnverwandten Verben, so $\mu \varepsilon \tau \alpha^{\prime}$ mit Gen.

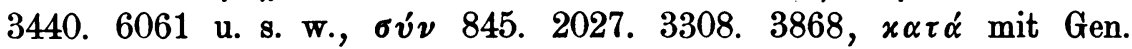

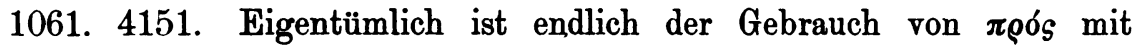

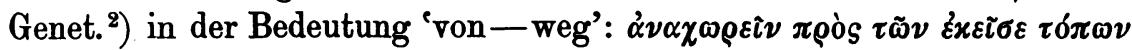
(5610, ähnlich 3621, und bei Verben wie $\lambda \alpha \mu \beta \alpha^{\prime} \nu \varepsilon \iota \nu, \mu \alpha \nu \vartheta \alpha^{\prime} \nu \varepsilon \iota \nu, \alpha i \tau \varepsilon i \nu$ u. ä.), von $\pi \varepsilon \rho i$ mit Dativ 'im Umkreis von' (3522), غ̇ंi mit Dativ 'in

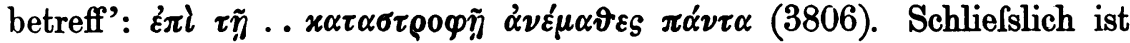

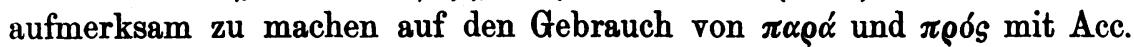
beim Superlativ in der Bedeutung 'vor'. Es hängt derselbe offenbar zusammen mit der Anwendung dieses Grades im Sinne des Komparativs $^{3}$ ); denn bei diesem war $\pi \alpha \alpha^{\prime}$ schon früh in dem Sinne von $\ddot{\eta}$

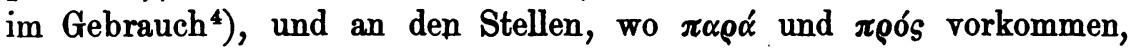
hat der Superlativ auch eine mehr komparative Bedeutung, nämlich 733:

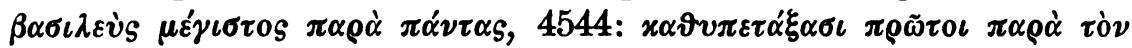

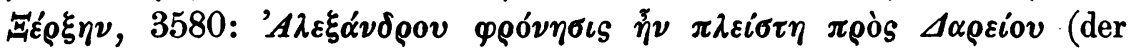

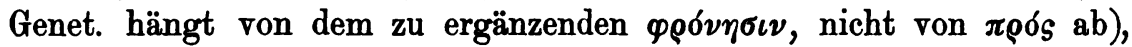
vgl. auch v. 717 .

Die Verwechslung oder Vertauschung der Ortsadverbia mit einander, die ja schon aus frühester Zeit stammt ${ }^{5}$ ), findet sich natürlich auch bei unserm Verf.; so kommt $\dot{\varepsilon} x \varepsilon \sigma \varepsilon$ für $\dot{\varepsilon} x \varepsilon \tilde{\imath} 30 \mathrm{mal}$ vor (z. B.

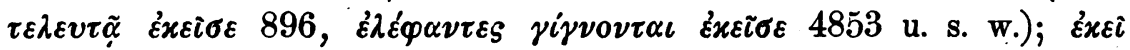
statt $\dot{\varepsilon} x \varepsilon i \sigma \varepsilon \varepsilon$ habe ich nur an zwei Stellen gefunden: 614.4833 ; charak-

1) Im Texte bei Wagner steht $\pi \rho 0 \sigma \varepsilon ́ \sigma \tau \eta$. Dafs dafür $\pi \rho \circ \varepsilon \sigma \tau \eta$ zu schreiben ist, vermute ich umsomehr, als in der Parallelstelle v. $1269 \delta \pi \rho \circ \alpha \sigma \theta \dot{\eta} \mu \varepsilon v o s$

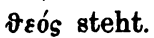

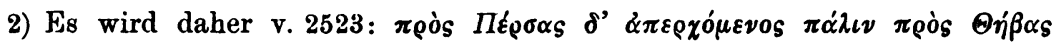

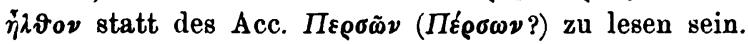

3) Man vgl. z. B. v. 3042, wo der Genetiv dabei steht wie sonst beim Kom-

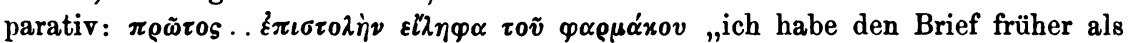

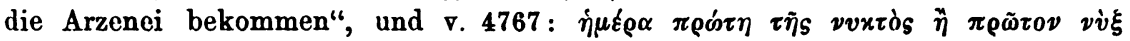

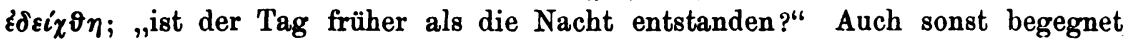

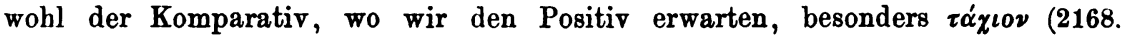
2944. 3039. 3251 u. s.), aber auch andere Wörter; man vgl. v. 250. 650. 3317. 4170. 4288; statt des Superlativs 404; der Positiv statt des zu erwartenden Komparativs 5579. Vgl. im allgem. Winer, Gr. S. $219 \mathrm{f}$.

4) Hatzidakis, Einl. S. 211. Vgl. Krüger, Gr. Spr. 49, 2, 8; Kühner, Gr. Gr. 2,445 f. 451.

5) Kühner, Gr. Gr. 2, 473 A. 4; Lobeck, Phryn. p. 127 A.; Winer 442 f.; Schmid, Att. 1, 91 f.; 2, 42 f.; 3, 58 f.; Hatzidakis, Einl. S. 210. 
teristisch ist auch v. 127, wo Nektanabus die ägyptischen Götter sieht:

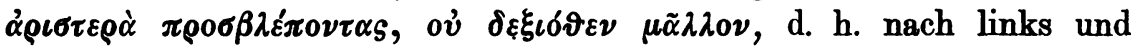
nicht nach rechts. So heifst ह̈ $\sigma \omega \vartheta \varepsilon \nu$ erstens 'hinein' (2234. 4958), aber auch 'drinnen' (5501), und in ähnlicher Weise werden $\pi \varepsilon \dot{\varepsilon} \alpha \vartheta \varepsilon v$ (für

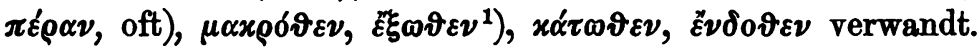

\section{Der Artikel.} und zwar

1) Der Artikel wird gesetzt, wo er früher nicht gebraucht wurde,

a) in ganz unmotivierter Wiederholung, wie $\dot{\varepsilon} x \quad \tau \tilde{\omega} \nu \stackrel{\alpha}{\alpha} \lambda \lambda \omega \nu \quad \tau \tilde{\omega} \nu$

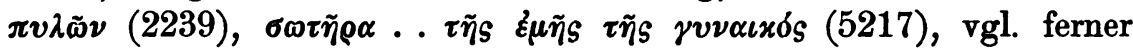
v. 902.3081 .4909 .5725 f. 6060.6082 ;

b) bei Adjektiven, wo er entweder sonst nicht stand oder jedenfalls das erstere attributiv gestellt wurde, so besonders bei Zahlbegriffen

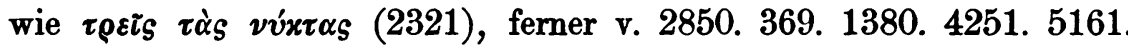
1527. 3375. 5026. 5406. 349. 4188; of $\pi \alpha^{\prime} v \tau \varepsilon s$ manchmal ganz gleich-

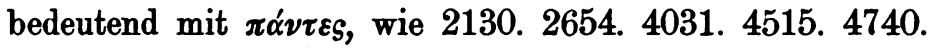

2) Der Artikel fehlt viel seltener; nur vereinzelt bei oṽzos, z. B. 470. 1687. 1736. 3681. 4945. 5607, und bei $\pi \tilde{\alpha} S$ in der Bedeutung 'ganz', z. B. 2967. 3310. 4324.

3) Die Stellung des Adjektivs beim Artikel und besonders des possessiven Genetivs ist ohne Unterschied bald attributiv, bald prädi-

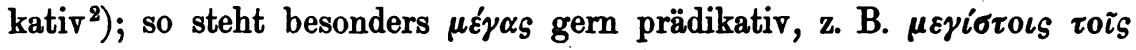

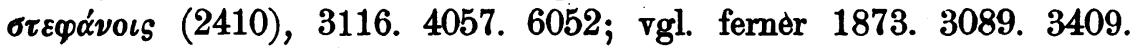
3883. Ganz besonders wird der possessive Genetiv $\alpha \dot{v} \tau 0 \tilde{v}, \alpha \dot{v} \tau \tilde{\nu} \nu$ bald attributiv, bald prädikativ gestellt; der Verf. sagt also z. B. rò $\nu$ $\chi \alpha-$

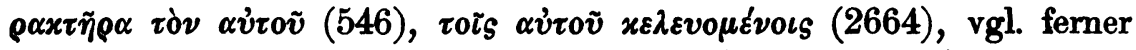
v. 2748. 3741. 4706 u. s.; seltener ist diese Stellung bei $z \mu o \tilde{v}$ u. s. w.,

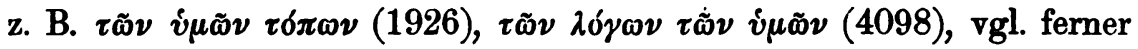
v. 2559. 3695. 3913. 3964. 5022. 5493. 5507 u. s., und noch seltener

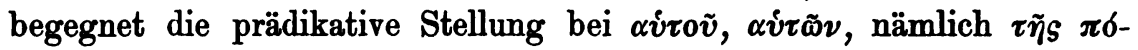

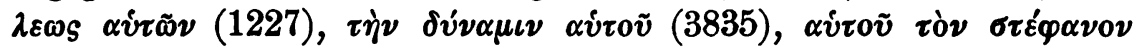

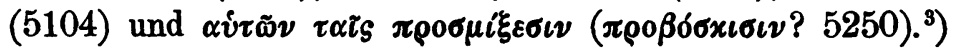

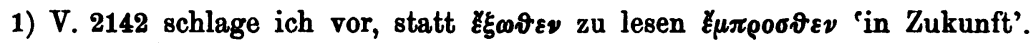

2) Vgl. Usener, D. heil. Theod. S. 148. Schmid, Att. 2, $48 \mathrm{f}$.

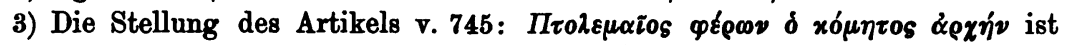
so eigentümlich, dafs man versucht sein könnte, einen Fehler des Abschreibers

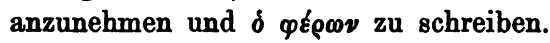




\section{Pronomina.}

Eine ganz aufserordentliche Vorliebe hat unser Verf. für das Pronomen $\alpha \dot{v} \tau$ ós. Er gebraucht es einmal allein stehend für 'er' und 'sie' an Stellen, wo zu einer Hervorhebung gar keine Veranlassung vorlag ${ }^{1}$ ), oder als unbegründeten Zusatz zu einem Partizip so oft, dafs ich von einer Angabe von Stellen Abstand nehme (man vgl. v. $388 \mathrm{f}$. 1707. 2074 f. 3543. 4437). Aufser dem einfachen av̉rós wird in derselben Bedeutung auch $\delta$ av่rós, wenn auch nicht in gleicher Ausdehnung,

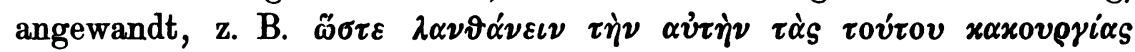

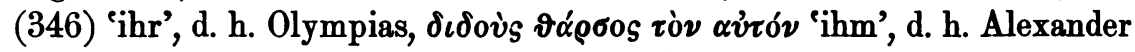
(2943), ferner v. 548. 3951. 4110. 2014 und 6061, eine Stelle, die ich ganz hersetze, weil ich eine Änderung der Lesart vorschlage:

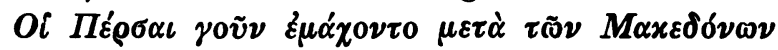

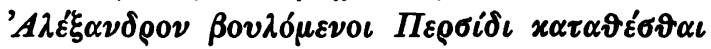

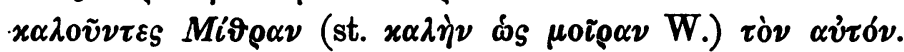

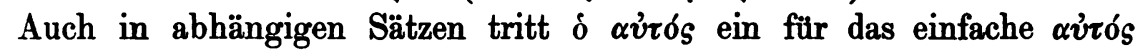

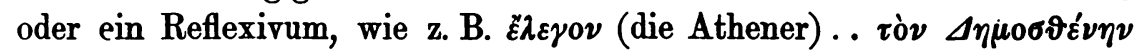

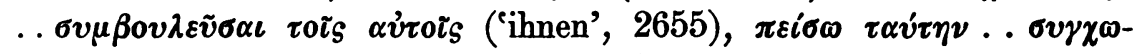

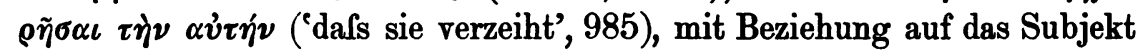

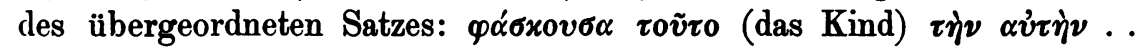

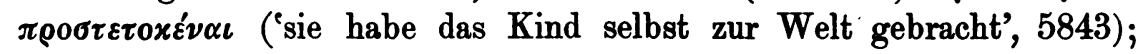
man vgl. ferner 418. 747. 1578. 1899. 1946. 2014. 3419. 4244. 4874.

Zweitens wird $\alpha$ v่rós zu Substantiven hinzugesetzt, ohne dafs etwa ein Gegensatz hervorgehoben werden soll, und zwar ohne und mit Artikel in prädikativer und attributiver Stellung.

1) Sehr oft erscheint $\alpha \dot{v} \tau \delta_{\xi}$ bei Eigennamen, z. B. bei 'A $A \varepsilon^{\prime} \xi \alpha \nu \delta \rho o s$

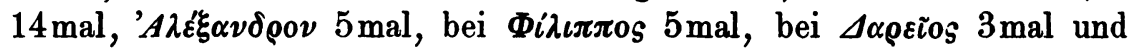

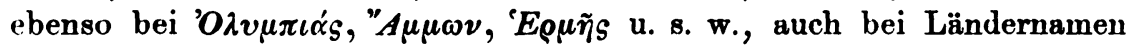

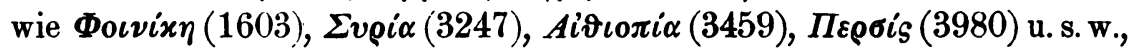

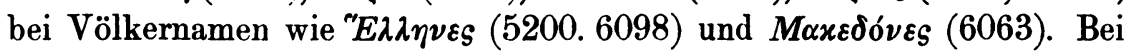
Appellativen tritt av่rós so oft hinzu, dals es häufig eigentlich völlig dèm Artikel entspricht, und auf jeder Seite eine Zahl von Beispielen vorkommt; ich trage, um eins anzuführen, die Stellen zusammen, wo

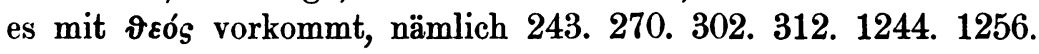

2) Mit dem Artikel wird es seltener in attributiver Stellung gebraucht, hat aber dann an diesen Stellen keineswegs die Bedeutung 'derselbe';

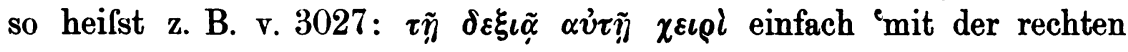

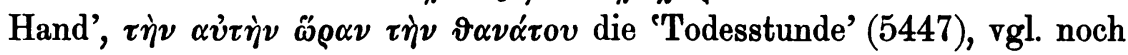
3750. 4142. Sehr viel ausgedehnter ist der Gebrauch von av่ròs $\delta$,

1) Hatzidakis, Einl. S. 208. 


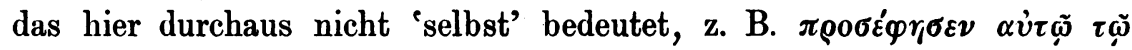

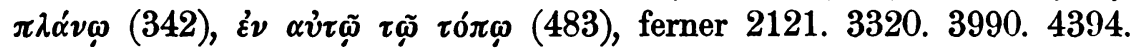
5954 und an sehr vielen andern Stellen. Auch zu Eigennamen tritt

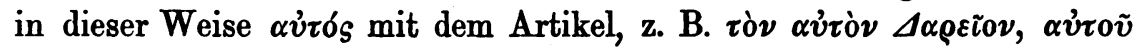

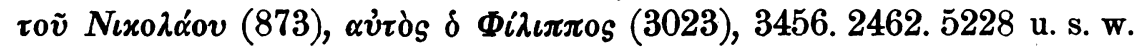

Bei dem Reflexivpronomen zeigt der Verfasser eine besondere Eigentümlichkeit; während nämlich die Beziehung von $\varepsilon \alpha v \tau o \tilde{v}, \varepsilon \alpha v \tau \tau \tilde{\omega} \nu$ auf die erste und zweite Person schon in klassischer und noch mehr in der späteren Zeit vorkam ${ }^{1}$ ) - und auch von unserm Verf. an-

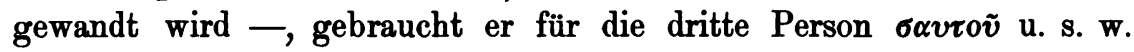

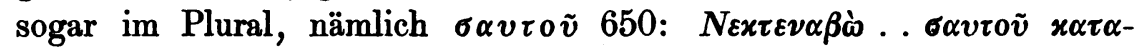

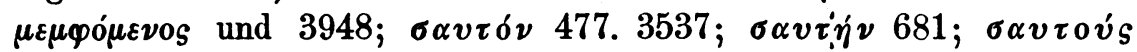
4511. 4650. Da dieser Gebrauch also feststeht, so vermute ich, dals

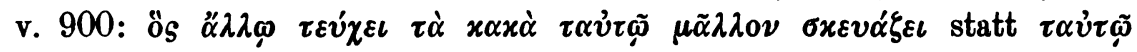
$\mathrm{zu}$ lesen ist $\sigma \alpha v \tau \tilde{\omega}$.

Für das Possessivpronomen der dritten Person, das sich auf das Subjekt desselben Satzes bezieht, gebraucht unser Verf. aufser-

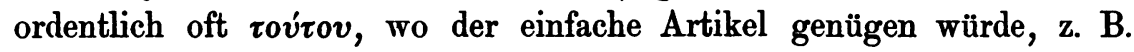

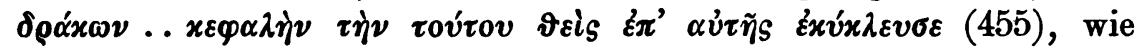
denn überhaupt die obliquen Kasus von oṽzos auch in abhängigen Sätzen auf das Subjekt des regierenden Satzes sich zurückbeziehen. Schwierig ist die Entscheidung über die Frage, wann der Verf. $\alpha \dot{v} \tau o \tilde{v}$, $\alpha \dot{v} \tau \tilde{\nu} \nu$ und $\alpha \dot{v} \tau o \tilde{v}$, $\alpha \dot{v} \tau \tilde{\omega} \nu^{2}$ ) für das Possessivpronomen, das sich auf das Subjekt bezieht, anwendet, da sehr leicht der Spiritus vertauscht, bezw. verlesen sein kann, wie denn thatsächlich auch an manchen Stellen Verschiedenheit der Lesungen zwischen Wagner und Legrand besteht. Im allgemeinen verwendet der Verf. $\alpha \dot{v} \tau o \tilde{v}$, $\alpha \dot{v} \tau \tilde{\omega} \nu$ bei Beziehung des Pronomens auf das Subjekt desselben Satzes, und zwar auch, wo das Pronomen nach früherem Sprachgebrauch fehlen könnte und

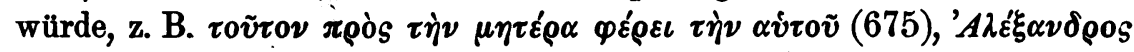

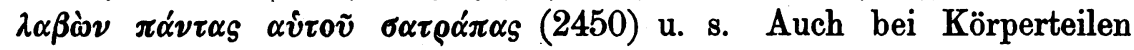

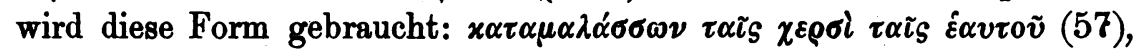
3588. 3880. 4995. 5250; ich vermute daher, dafs v. 61. 3673. 5249

1) Hatzidakis, Einl. S. 189.

2) Was die Form anbelangt, so ist die überwiegende durchaus $\alpha \dot{v} \tau \tilde{v}$ u. s. w.,

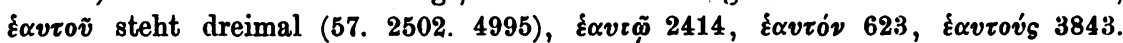

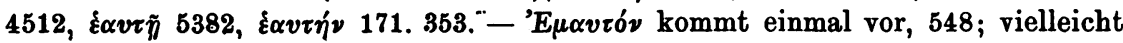
einmal auch $\xi \mu_{0} \tilde{v} \alpha \tilde{v} \tau \tilde{\eta} s$, wenn, wie ich vermute, v. 633 statt $\delta \mu_{0} \tilde{v} \tau^{\prime} \alpha \hat{v} \tau \tilde{\eta} s$ zu lesen ist $\dot{\varepsilon} \mu 0 \tilde{v} \tau^{\prime} \alpha \dot{v} \tau \tilde{\eta} s$. Es müfste sonst $\alpha \dot{v} \tau \tilde{\eta} s$ reflexiv gleich $\xi_{\mu \alpha} \mu v \tilde{\eta} s$ gebraucht sein, was allerdings nicht unmöglich ist, da $\alpha \dot{v} \tau \tilde{\eta} s$ auch einmal in dem Sinne

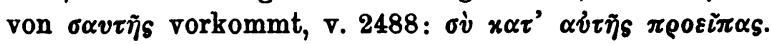


statt des dort stehenden $\alpha \dot{v} \tau o \tilde{v}$, bezw. $\alpha \dot{v} \tau \tilde{v} v$, zu lesen ist $\alpha \dot{v} \tau o \tilde{v}$, bezw. $\alpha \dot{v} \tau \tilde{\omega} \nu$. Nur selten kommt es vor, dafs $\alpha \dot{v} \tau o \tilde{v}, \alpha \dot{v} \tau \tilde{\omega} \nu$ nicht auf das

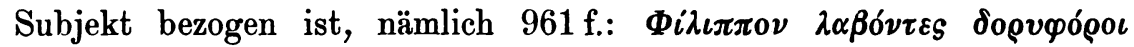

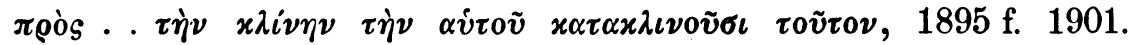
5807. 5942; ich vermute, dafs jedenfalls an den drei letzten Stellen $\alpha \dot{v} \tau o \tilde{v}$ und $\alpha \dot{v} \tau \tilde{\omega} \nu$ zu lesen ist. Sehr viel häufiger begegnet es dagegen, dafs $\alpha \dot{v} \tau o \tilde{v}, \alpha \dot{v} \tau \tilde{\omega} \nu$ auf das Subjekt bezogen werden, abgesehen von den eben besprochenen an 13 Stellen; ich bemerke dazu nur, einmal, dafs in den beiden Versen, wo auch die Lesungen Legrands vorhanden sind,

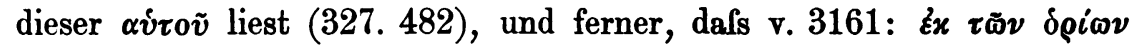

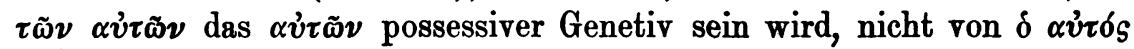
abzuleiten ist.

In betreff der Relativpronomina bemerke ich zunächst, dafs

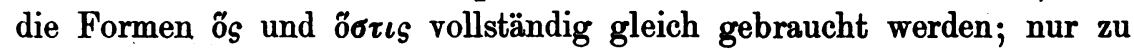
Anfang eines neuen Satzes, wo der Verf. nach lateinischem Vorbilde die relativische Anknüpfung liebt, sind die Formen von ös gebräuch-

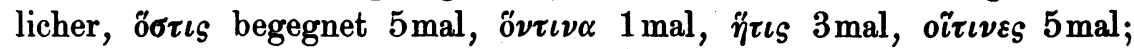

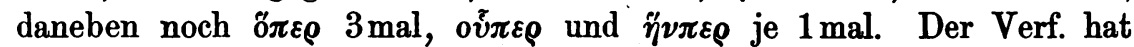
ferner eine Vorliebe für Voranstellung des Relativs und Hereinziehung

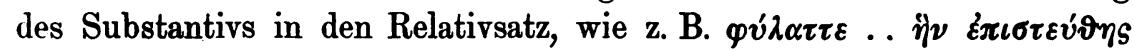
$\chi \omega ́ \varrho \alpha \nu$ (107), vgl. 938. 2100 u. s., ebenso wendet er die Assimilation

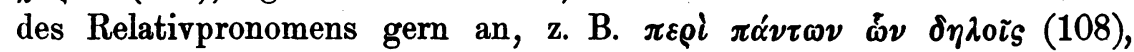

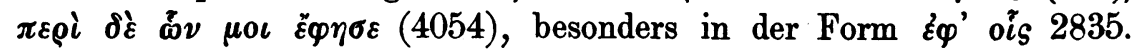
3186. 3815, sodafs schliefslich '́s' oís fast vollständig in die Bedeu-

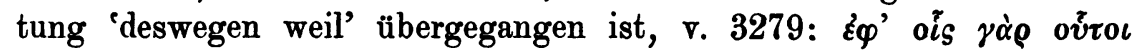

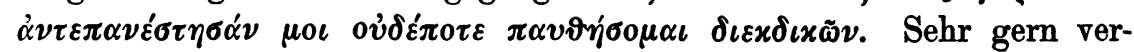
bindet der Verf. mit einem solchen Relativum ein Substantivum, und

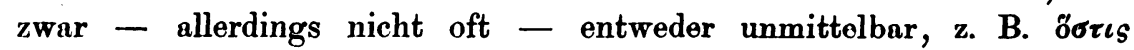

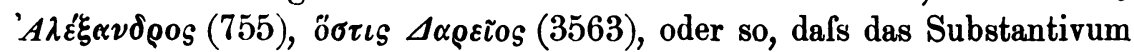
nach einem Partizip oder einer Verbalform das Relativ wieder auf-

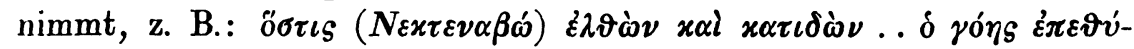

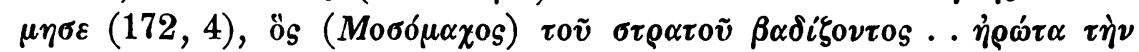

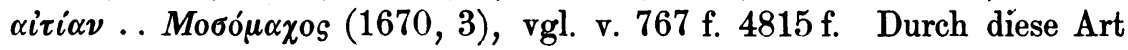
und die Vorliebe für relativische Anknüpfung ist der Verf. dann weiter dazu gekommen, das Relativ an den Anfang ohne jede Beziehung zu setzen und dann entweder das Substantiv hinzuzufügen, wie: $\left.{ }^{\circ} s^{1}\right) \dot{\varepsilon} v$

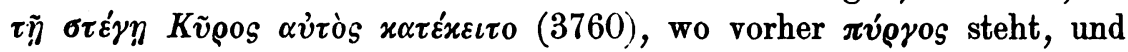

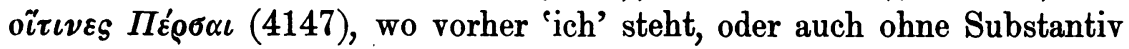

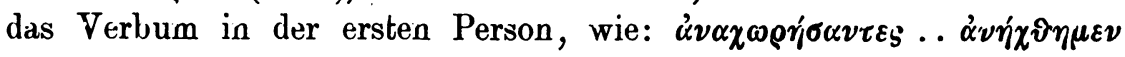

1) Vielleicht ist. hier ov statt ôs zu lesen. 


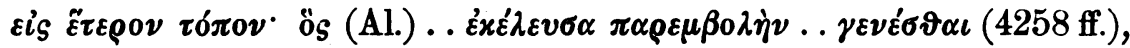
vgl. 182. $4424 \mathrm{f}$. Viel häufiger aber wird zu dem Relativum noch ein

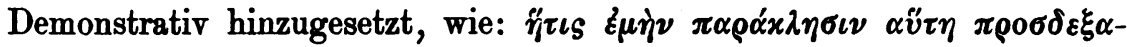

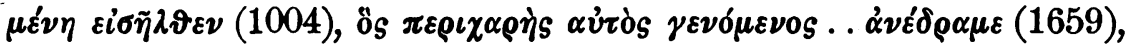
vgl. ferner v. 438 f. $743.1108,10.2264,6.2479$ f. 2485 f. 3130. 3427 f. 3559 ff. 4430 f. 4521 f.

Die Anwendung des Interrogativpronomens $\pi 0 i s^{1}$ ) für das einfache $\tau$ is begegnet auch bei unserm Verf. ${ }^{2}$ ), aber nur an Stellen, wo auch die Vorlage dasselbe bietet, und auch die von $\varepsilon i_{\text {í }}$ für das indefinite Pronomen $\tau \iota$, aufser an den mit der Vorlage übereinstimmenden Stellen noch v. 1636. 3125. 3385. 4876. Umgekehrt erscheint manchmal $\mu \dot{y}^{\prime} \tau \iota s$ oder auch $\tau \iota \varsigma \mu \eta^{\prime}$ statt des sonst gewöhnlichen $\mu \eta \delta \varepsilon i_{S}$, so

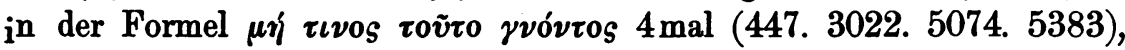

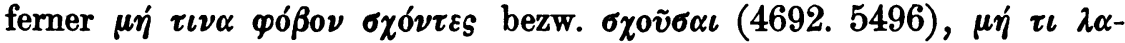

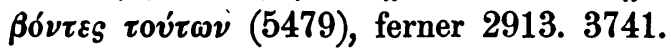

\section{Ver bum.}

\section{a. Genera.}

Manchmal verwendet der Verf. das Aktiv, wo sonst gewöhnlich

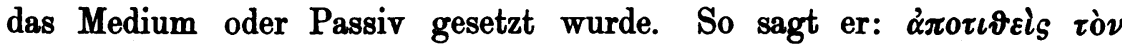

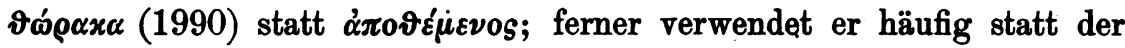
gebräuchlichen Umschreibung des einfachen Verbums durch ein Sub-

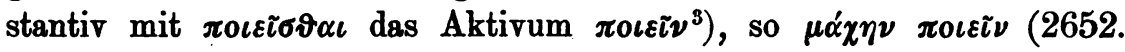

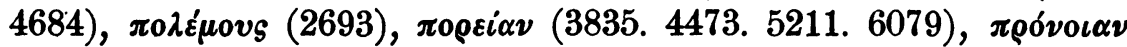

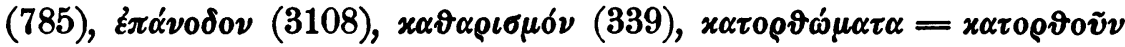

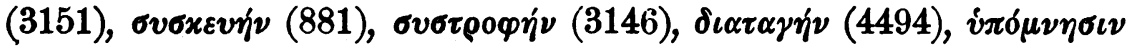
(4496); selbst $\dot{\mu} \mu \pi 0 \iota \varepsilon \tau \nu$ wird in dieser Verbindung gebraucht: $\dot{v} \lambda \alpha x \dot{\nu} \nu$

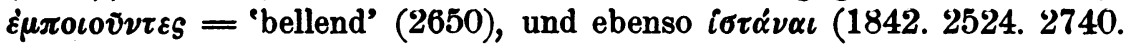
5127. 5495. 845). Hierher gehört es auch, wenn der erste Aorist

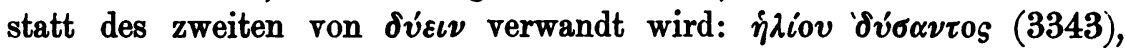

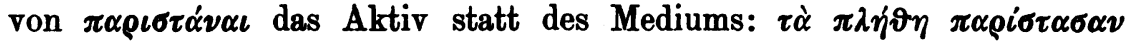

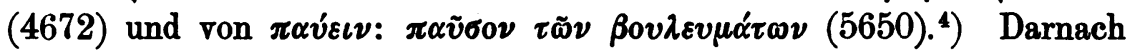

1) Vgl. Hatzidakis, Einl. S. 207.

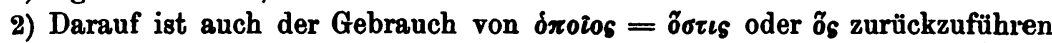

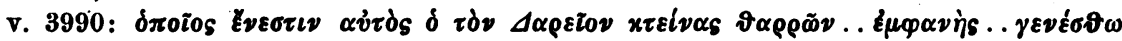

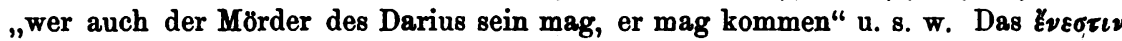
ist völlig gleichbedeutend mit żoriv, wie im N. T. हैvı (8. Hatzidakis, Einl. S. 207).

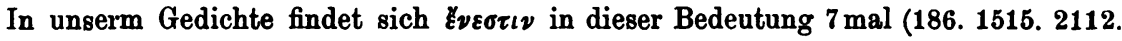

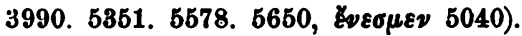

3) Vgl. Hatzidakis, Einl. S. $197 \mathrm{f}$.

4) Kühner, Gr. Gr. 2, 83. 84. Hatzidakis, Einl. S. 202. 
sind auch die Formen

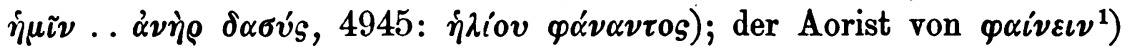

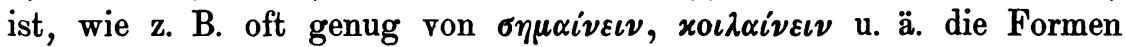
$\dot{\varepsilon} \sigma \eta \dot{\eta} \mu \nu \alpha$ (in Bi öfters, z. B. 1871) und $\dot{\varepsilon} x o i \lambda \alpha \nu \alpha$ (4679) vorkommen, $\left.\ddot{\varepsilon} \varphi \alpha \nu \alpha^{2}\right)$ gebildet, und dies in neutraler Bedeutung gebraucht, weil die

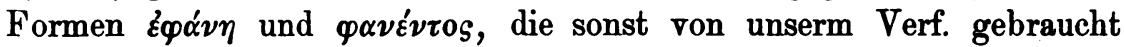
werden, wegen des Accentes nicht in den Vers gingen.

Zweitens zeigt der Verf. eine Vorliebe für das $\mathrm{Medium}^{3}$ ) von manchen Verben, ganz besonders im Infinitiv, so besonders $\lambda \alpha \beta \varepsilon \sigma^{\prime} \vartheta \alpha \iota$ (1253. 1925. 2183. 2435 u. s.) und die Komposita $\pi \rho 0 \sigma \lambda \alpha \beta \varepsilon \sigma_{\sigma} \sigma \alpha \iota$

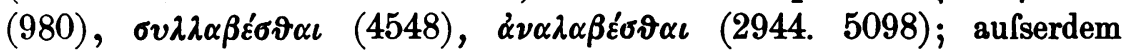

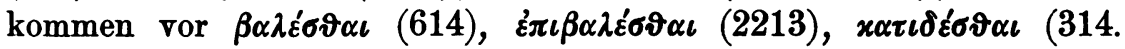

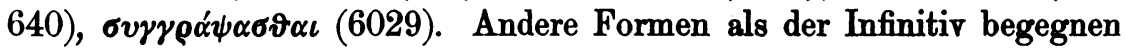

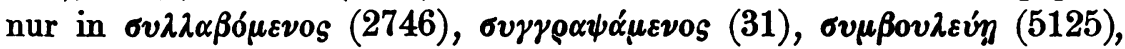
$\beta 0 v \lambda \varepsilon v i \eta n$ (2945) und $x \alpha \tau \alpha \sigma x \alpha \psi \delta \mu \varepsilon v \alpha(1513$, st. $x \alpha \tau \alpha \sigma x \alpha \varphi \delta \mu \varepsilon v \alpha$ W.) als Futurum Passivi.

Endlich wird bisweilen der passive Aorist statt des sonst gebräuchlichen medialen gesetzt, bezw. werden beide Formen in ganz gleicher Bedeutung angewandt. So $\pi \alpha v \vartheta \eta^{\sigma} \sigma \mu \alpha \iota$ (3280, 'ich werde aufhören'),

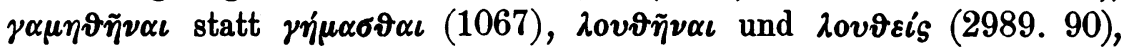

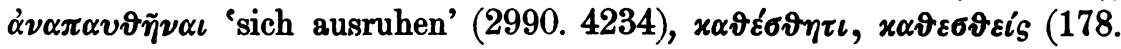

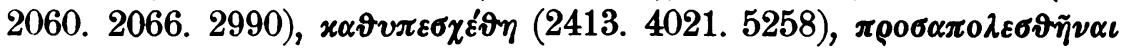

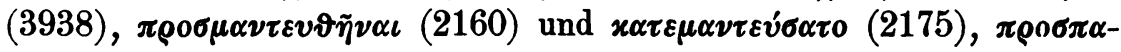
$\rho \alpha \tau \alpha^{\prime} \xi \alpha \sigma \vartheta \alpha \iota(2621), \alpha^{2} \nu \tau \iota \pi \alpha \rho \alpha \tau \alpha \chi \vartheta \tilde{\omega} \mu \varepsilon \nu(2975.3731), \pi \alpha \rho \alpha \tau \alpha \chi \vartheta \hat{\vartheta} \nu \tau \varepsilon \mathcal{S}(1560)$.

\section{b. Tempora.}

Nicht selten wendet der Verf. statt des Aorists das Präsens, Imperfekt, Perfekt oder Plusquamperfekt an, und zwar das Präsens z. B.: $\xi \vartheta \alpha \psi \varepsilon \nu . ~ . ~ \varkappa \alpha i ~ \varkappa \alpha \tau \alpha \tau i \vartheta \eta \sigma \iota \nu ~(684,6)$, ferner 647, 8. 1030.

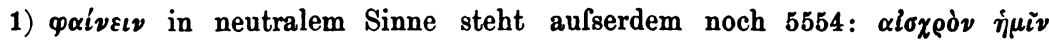

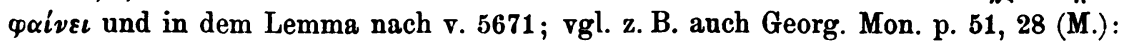
jiaiov saivovios.

2) Vgl. Lobeck, Phryn. p. 26; Schmid, Att. 1, 229; 2, 24; 3, 35; Hatzidakis, Einl. S. 202. 286; Usener, D. heil. Theod. S. 138. Die Form Évย́q $\alpha \nu \varepsilon$ in transitiver Bedeutung findet sich auch in der Hs L des Ps.-K. 1, 13 p. 714 A. 17:

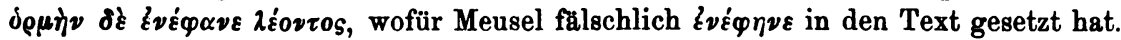

3) Darnach wird wohl die Form $\delta \eta \lambda_{0} \tilde{\tau}{ }^{\prime}$ (v. 408 nach Legrand, $\delta \eta \lambda_{0} \tau^{\tau} \tau^{\prime}$ W.) $=\delta \eta \lambda_{0} \tau \alpha \iota$ als Medium mit aktiver Bedeutung zu fassen sein, von dem Infinitiv $\delta \eta \lambda_{0 i \tau}$ (Hatzidakis, Einl. S. 193). Mit Rücksicht auf diesen Gebrauch schlage ich 70:, v. 1584 statt des unverständlichen $\sigma \dot{\jmath} r \alpha \tau \alpha \pi \alpha \tau \dot{j} \sigma \alpha \nu \tau \alpha$ zi lesen $\sigma \grave{c} x \alpha \tau \alpha-$ $\pi \alpha \tau \eta \dot{\sigma} \sigma \alpha \sigma \vartheta \alpha \iota$, sodafs der mediale Aorist statt des sonst gebräuchlichen aktiven stehen würde. 
1882. 2091. 3205. 3560. 5320 u. s. ${ }^{1}$ ); das Imperfekt v. 1771. 2154. 2249. 5376 u. s. Zahlreicher sind die Stellen, wo Perfekt und Plusquamperfekt eintreten statt des erwarteten Aorists, besonders von $\lambda \varepsilon \dot{\gamma \varepsilon \iota \nu}$ (260. 732. 2609. 2962. 3037. 3751. 4168. 5413. 5287. 376),

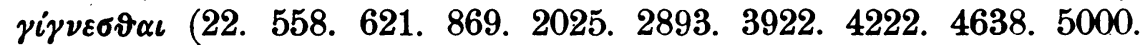
5759. 3218. 4093. 4596. 4643. 5253. 5928, vgl. auch den Infinitiv

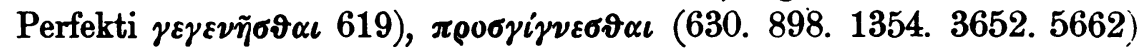

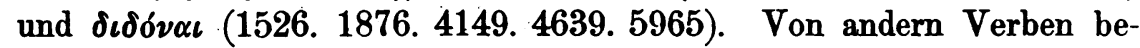
gegnen die Formen $\check{\varepsilon} \gamma \nu \omega x \varepsilon \nu$ (5907), $\delta \varepsilon \delta \dot{\eta} \lambda \omega x \varepsilon$ (5883), $\varepsilon i \sigma \varepsilon \lambda \dot{\eta} \lambda v \vartheta \varepsilon$ (4519), "̌б $\chi \eta x \varepsilon$ ('er gewann' 1532, 'er besafs' 4668), x'́x $x \lambda \chi x \varepsilon$ (1181),

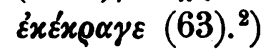

Eine Eigentümlichkeit ist ferner, dafs unser Verf. das Imperfekt und auch den Aorist nicht ganz selten durch Umschreibung mit $\eta^{\nu} v$

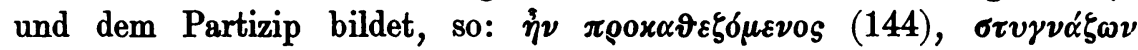

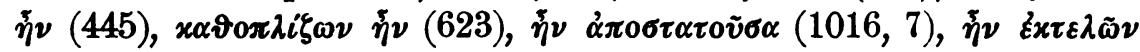
(1075), 光

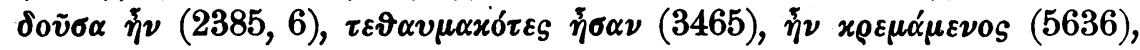

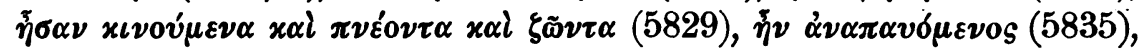

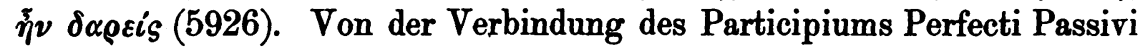
mit $\varepsilon^{i} \nu \alpha \iota$ oder $\left.\tilde{\varepsilon} \chi \varepsilon \iota \nu^{3}\right)$ habe ich dagegen nur zwei Beispiele gefunden: $\delta \varepsilon \delta 0 v \lambda \omega \mu \varepsilon \dot{v} \eta \eta$ oṽ $\sigma \alpha(6024)$ und $\varepsilon^{i} \chi \varepsilon \dot{\eta} \gamma \alpha \pi \eta \mu \varepsilon \dot{\varepsilon} \eta \nu$ (3543).

\section{c. Modi.}

Die Anwendung des einfachen Optativs für den Potentialis ${ }^{4}$ ) begegnet auch bei unserm Verf. sowohl in Bedingungssätzen (z. B. 724 . 3719), wie auch in der Behauptung (z. B. 1054. 1457. 2779), und

1) Darnach mőchte ich v. 503 ff. die Tempora, die schwerlich richtig sein konnen, andern. Der Wabraager deutet das dem König Philipp zu teil gewordeno Wunderzeichen von der Schlange und dem Ei und sagt:

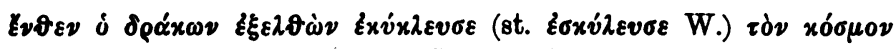

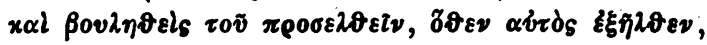

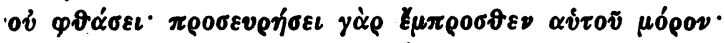

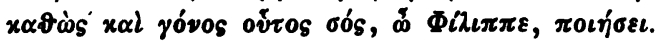

Offenbar mufs v. 505 noch auf die Schlange bezogen werden, denn die Anwendung auf Alexander kommt erst im nächsten Vers; ich schlage daher vor zu lesen:

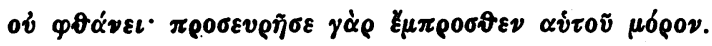

Den Accent kann ich zwar aus diesem Gedichte nicht belegen; er ist aber durchaus möglich.

2) Kühner, Gr. Gr. 2, 129 A. 2; Schmid, Att. 1, 95; 2, 52; Hatzidakis, Einl. S. 204 f. Vgl. auch Usener, D. heil. Theod. S. 120.

3) Hatzidakis a. a. 0 .

4) Vgl. Kühner, Gr. Gr. 2, 191; Schmid, Att. 1, 244. 
ebenso erscheint für den Irrealis ein historisches Tempus ohne ${ }_{\alpha} \nu^{1}$ ) (z. B. 1223. 3986. 4437). Auch der Konjunktiv in imperativischem Sinne findet sich an manchen Stellen, und zwar sowohl prohibitiv (Konjunktiv Praesentis), wie auffordernd ${ }^{2}$ ) (z. B. 1230. 1548 f. $\left.^{3}\right) 1976$. 4000. 5060. 5310). Noch viel ausgedehnter aber ist der Gebrauch des Konjunktivs in futurischem Sinne von einer grofsen Anzahl von Verben $^{4}$ ), besonders von yi

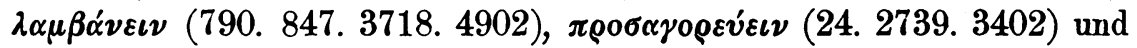
zahlreichen andern. Daher wird denn erstens in abhängigen Sätzen der Konjunktiv vollständig dem Futurum gleichgesetzt (wie $1795 \mathrm{ff}$.:

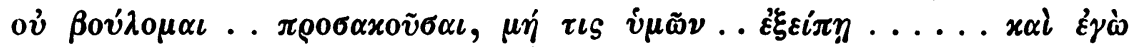

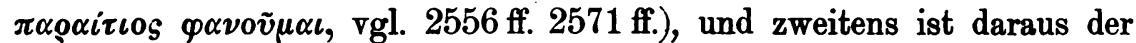
Konjunktiv in abhängigen Frage- und Aussagesätzen zu erklären (wie

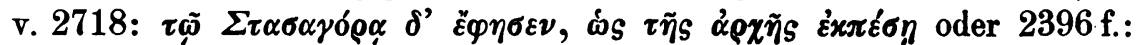

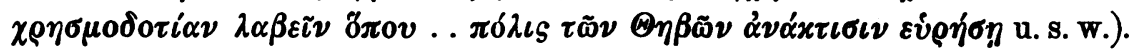

Willkür und Unklarheit zeigt der Verf. bei andern Konjunktionen

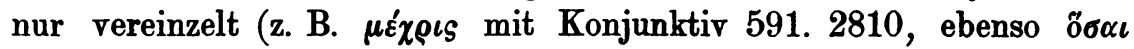

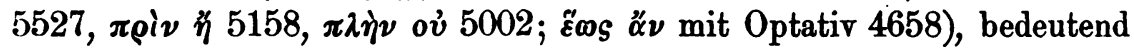
mehr dagegen, wie die spätere Gräzität überhaupt, in Bedingungssätzen. Einmal werden in ganz auf derselben Stufe stehenden Bedingungssätzen verschiedene Konjunktionen und Konstruktionen angewandt, wie 1675 ff.:

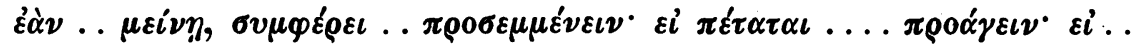

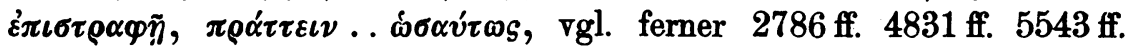
$6031 \mathrm{ff}$. Zweitens steht in Sätzen mit $\varepsilon i$ der Konjunktiv ${ }^{5}$ ) (212 ff. 244.

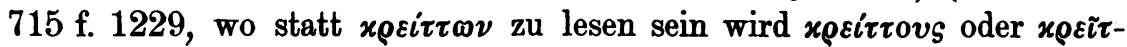
$\tau o v, 1730$. 1980. 3311. 3791. 3843. $\left.3976 \mathrm{f}^{6}{ }^{6}\right) 5215$ ff. 5534. 5540. 5771.

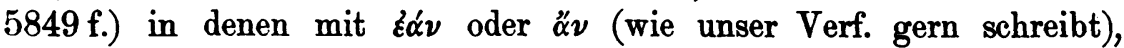
wenn auch seltener, der Indikativ (1241 f. $2976 \mathrm{ff}$. $3192 \mathrm{ff}$., wo für $\varepsilon i$ wohl $\eta^{\nu} \nu$ geschrieben werden mufs, $3500 \mathrm{f}$.).

1) Vgl. Winer a. a. O. S. 280 f.; Hatzidakis, Einl. S. 219.

2) Hatzidakis, Einl. S. 219.

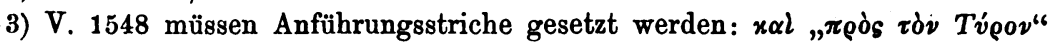

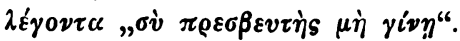

4) Vgl. Lobeck, Phryn. 722 ff.; Kühner, Gr. Gr. 2, 184; Mullach a. a. 0. S. 361; Hatzidakis, Einl. S. 218 f. Unser Verf. gebraucht auch den Opt. gleichbedeutend

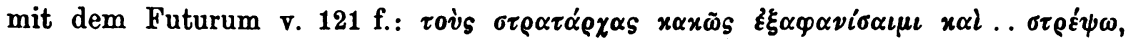
ferner 507. 3817; Optativ und Konjunktiv zusammen in futurischem Sinne v. $279 \mathrm{f}$.

5) Vgl. Kühner, Gr. Gr. 2, 207; Schmid, Att. 1, 244; Winer a. a. 0. S. 270 f.

6) Die Verse $3976 \mathrm{f}$. scheinen in der Wagnerschen Fassung keinen Sinn zu geben; ich vermute, daîs zu lesen ist:

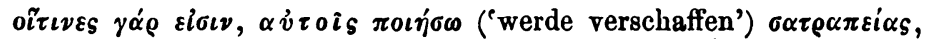

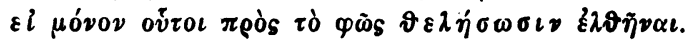


Nur vereinzelt begegnen wir dagegen der im Vulgärgriechischen allgemein gebräuchlichen Umschreibung des Infinitivs' ${ }^{1}$ ) mit ï $\nu \alpha$, bezw. ö $\pi \omega s$; ersteres erscheint nur einmal bei $\beta o v ́ \lambda \varepsilon \sigma \vartheta \alpha \iota$ und zwar zusammen

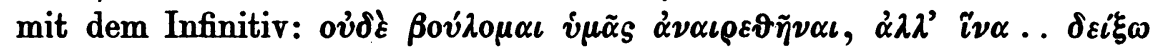

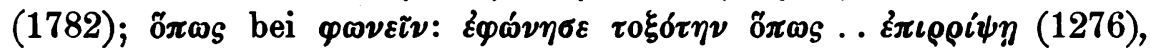

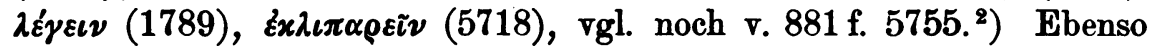
vereinzelt begegnet der Konjunktiv in abhängigen Wunsch- und Auf-

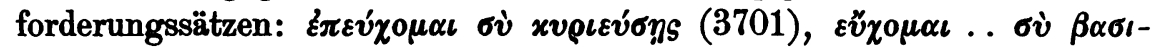

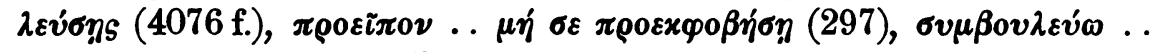

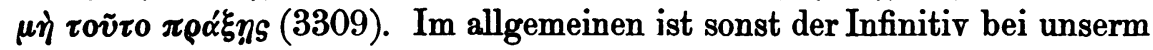
Verf. durchaus beliebt, sodafs er ihn sogar, vielleicht eben im Gegensatz zu dem Sprachgebrauch des Volkes, wo der Infinitiv immer mehr im Schwinden war, häufiger benutzt, als es früher der Fall war. Ein-

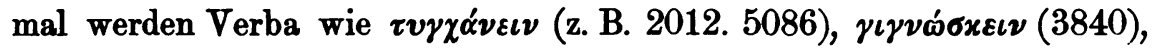
$\delta \varrho \tilde{\alpha} \nu$ (84f.) mit dem Infinitiv statt mit dem Partizip verbunden ${ }^{3}$ ),

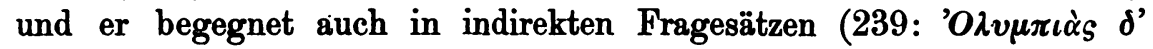

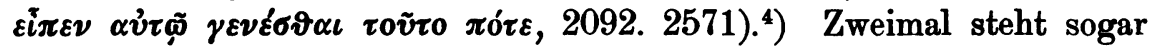
der Infinitiv mit $\dot{\omega}$ (bei $\gamma \iota \gamma \nu \omega ́ \sigma x \varepsilon \iota \nu 1247 \mathrm{f}$. und $\partial \mu \nu v ́ v \alpha \iota 1978 \mathrm{f}$ ), denn in den Sätzen, wo scheinbar sonst noch ம́s beim Infinitiv steht, ist derselbe wohl anders anfzufassen. Unser Verf. hat nämlich eine so entschiedene Vorliebe für diese Partikel, dafs er z. B. ö $\tau \iota$ in dem ganzen Gedichte nur $11 \mathrm{mal}$ anwendet, $2 \mathrm{mal}=$ 'weil' $\left.(1002.5420)^{5}\right)$, sonst nach Verben des Sagens u. ä. (194. 400. 1049. 1867. 2649. 2713. 3046. 3713. $3768=$ Zon. 4, 14 p. 349 B.), wozu noch eine Stelle kommt, in der $\dot{\omega}_{\mathcal{S}}$ ö $\tau \iota$ zusammen gebraucht ist $\left.(2722)^{6}\right)$, während sonst in derartigen Sätzen immer ம́s erscheint. Aufserdem aber verwendet es unser Verf., abgesehen von der gewöhnlichen Bedeutung 'wie', auch

1) Vgl. Hatzidakis, Einl. S. 214 f.

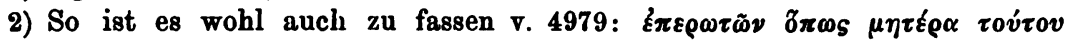

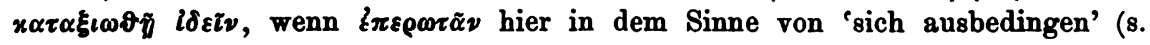
Sophocles, Gr. Lex. s. v.) gefafst wird; und diese Bedeutung ist wohl notwendig

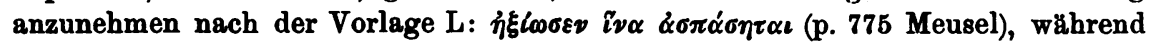

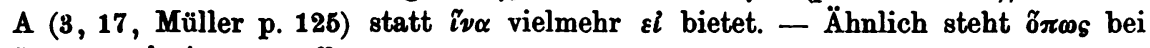
$\varepsilon_{\nu \varepsilon \sigma \tau \iota}=\xi \sigma \tau i$ v. $1515 \mathrm{ff}$.

3) Vgl. Hatzidakis, Einl. S. 227. - Partizip und Infinitiv zusammen ab-

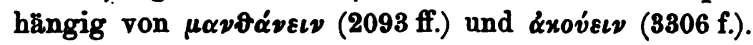

4) Vgl. Usener, D. heil. Theod. S. 145. - Auch v. 2162 , falls nicht statt

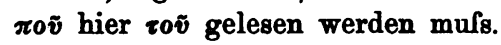

5) In dem Wagnerschen Texte ist allerdings ö $\tau \iota$ als Kolon gefafst, es ist

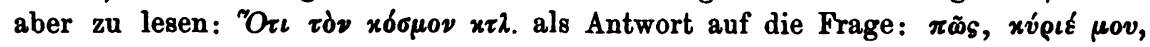
甲९র́rov.

6) Vgl. Hatzidakis, Einl. S. 213. 


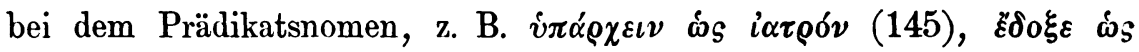

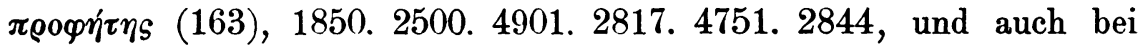

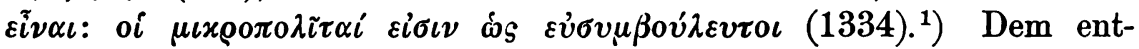
sprechend sind auch die Stellen zu erklären 2178 f.: $\pi \rho \circ \mu \eta \nu v ́ \omega \nu$ ஸs $\delta v \nu \alpha \tau o ́ v \sigma \varepsilon \ldots \gamma \varepsilon v \varepsilon \dot{\sigma} \sigma \alpha \iota, 3980$ und 5232, d. h. $\dot{\omega} s$ gehört nicht etwa zum Infinitiv, sondern zum Prädikatsnomen.

Besonders häufig wird der einfache Infinitiv gesetzt zur Bezeich-

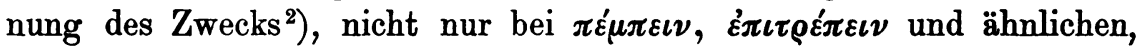

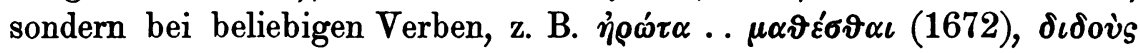

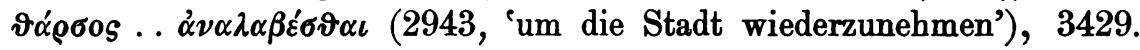
3687 u. s. w. Eine sehr beliebte Form ist der Infinitiv mit $\tau o \tilde{v}^{3}$ ) zur

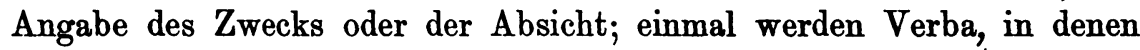

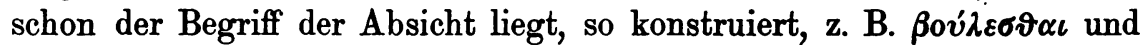
$\xi^{\prime} \vartheta \dot{\varepsilon} \lambda \varepsilon \iota \nu$ (sehr oft, z. B. 504. 720. 1090. 2788 u. ö.), ferner $\varepsilon \pi \iota \vartheta v \mu \varepsilon \check{\nu}$ (174), $x \alpha \vartheta \iota x \varepsilon \tau \varepsilon v \varepsilon \iota \nu$ (4516), $\mu \varepsilon^{\prime} \lambda \lambda \varepsilon \iota \nu$ (2440), $\pi \alpha \rho \alpha \chi \omega \rho \varepsilon \check{\nu}(5563), \pi \varepsilon i \vartheta \varepsilon \iota \nu$ (5367), selbst $x \varepsilon \lambda \varepsilon v \varepsilon \iota v$ (2205. 4695); ferner aber auch beliebige andere Verba, wie है $\rho \chi \varepsilon \sigma \vartheta \alpha \iota$ und seine Komposita (z. B. 693. 2418. 2596 f. 2789; vgl. ferner $3971 \mathrm{f}$. 6034). Dafs dabei dem Verf. die Auffassung für die Konstruktion völlig abhanden gekommen war, beweist der Satz:

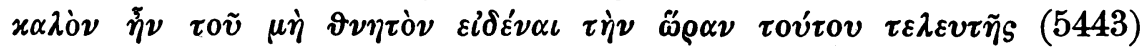
und der ähnliche v. 2792.

Das Partizip verwendet der Verf. zweimal substantivisch und

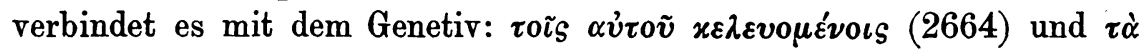

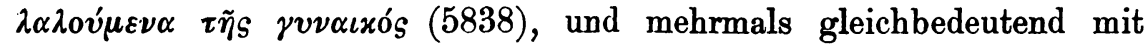
einem Adjektiv das Participium Perfecti, nämlich: $\tau \varepsilon \dot{x} x$ vov $\pi \varepsilon \varphi \iota \lambda \eta$ -

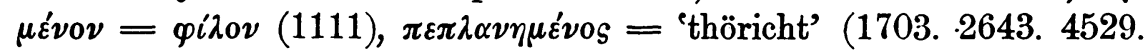

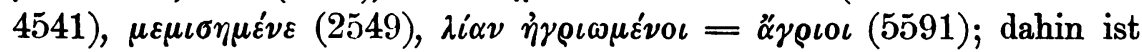
wohl auch die Form $\delta \beta \varepsilon \beta \alpha \sigma \iota \lambda \varepsilon v x \omega ́ s(4514)$ - 'der König' zu ziehen. $\left.{ }^{4}\right)$ Aufserdem wendet der Verf. es manchmal nach Verben an, wo

1) Darnach werden auch die Stellen zu erklären sein, wo scheinbar $\dot{\omega} s$ beim

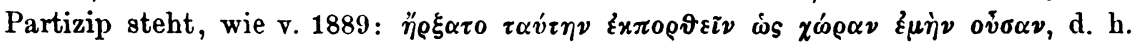

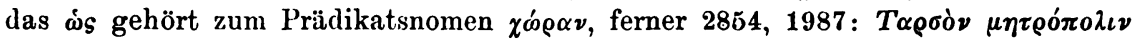
$\dot{\omega} s$ ov่ $\sigma \alpha \nu$, wo $\dot{\omega} s$ eigentlich ror dem Substantiv stehen mufste; aber eine solche

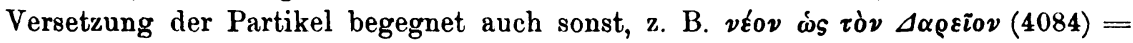

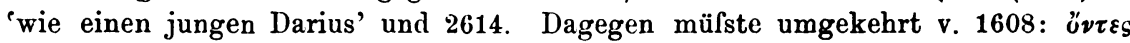

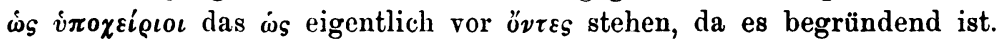

2) Vgl. Schmid, Att. 1, 97, 142; 2, 56; 3, 79. Hatzidakis in Kuhns Ztschr. $31,144$.

3) Krüger, Gr. Spr. 47, 22, 2; Kühner, Gr. Gr. 2, 604; Schmid, Att. 1, 90; 2, 40; Winer a. a. O. S. $299 \mathrm{ff}$; de Boor, Ind. zu Theoph. chron. II, 751 ; Sophocles, Gr. lex. Introd. p. 45.

4) Vgl. Schmid, Att. 1, 49. 240; 2, 53.

Byzant. Zeitschrift VII 2. 


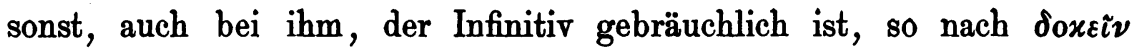

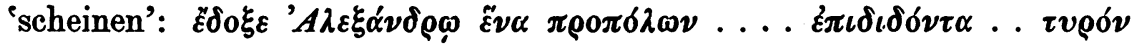

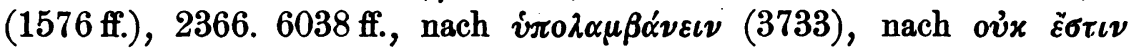

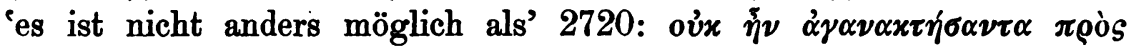

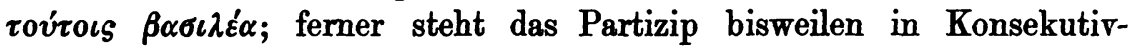

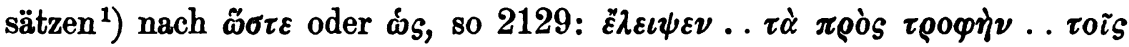

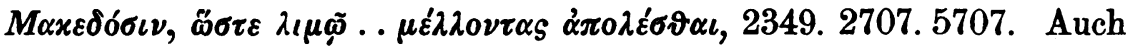
hier kommt die Konstruktion mit dem Infinitiv und dem Partizip

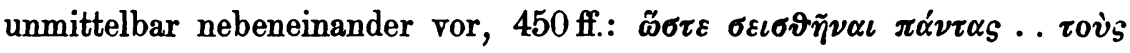

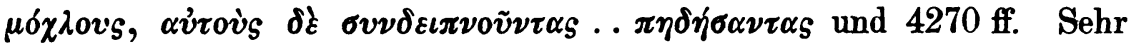
frei schaltet unser Verf. mit den eigentlichen Partizipialkonstruktionen, wie dies ja schon in früher Zeit vorkam ${ }^{2}$ ), später dann aber immer mehr überhand nahm.

1. $\mathrm{Zu}$ einem absoluten Genetiv wird noch ein Verbum finitum

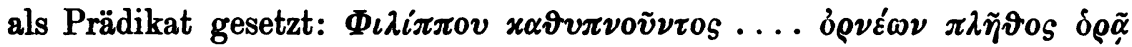
(481 f.), 509 ff. 3867 f. 4363 f. 5644 ff. 5759.

2. Sehr viel häufiger ist die umgekehrte Erscheinung, dafs nach dem Subjekt mit einem Partizip ein neues Subjekt gesetzt wird, d. h. also die gewöhnlich absoluter Nominativ genannte Konstruktion eintritt ${ }^{3}$ ),

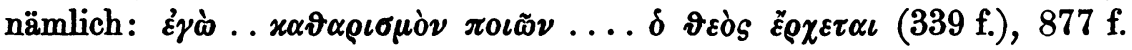
1201, 2. 1415 ff. $1591 \mathrm{ff} .1608$ ff. $) 1917$ f. 2464 f. $2477 \mathrm{ff} .2729$ f.

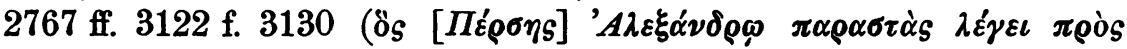

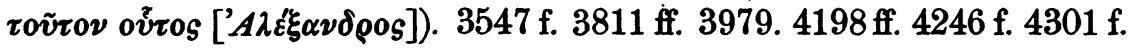
4338 ff. 4416.4678 f. 4914 f. 5111 f. 5150.5574 (wo Kapp a. a. 0.

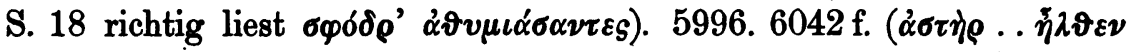

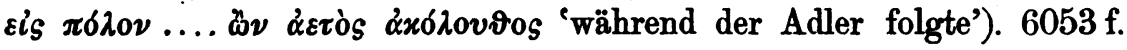
Hierher gehört auch v. $4430 \mathrm{ff}$.:

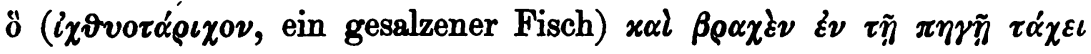

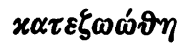

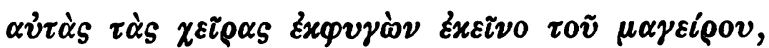

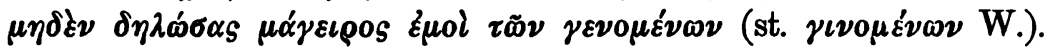

Schliefslich ist darnach auch zu erklären v. $4888 \mathrm{f}$.:

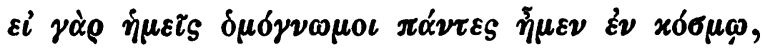

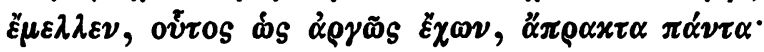

1) Vgl. dazu Kühner, Gr. Gr. 2, 1015 A. 3.

2) Kühner, Gr. Gr. 2, $661 \mathrm{ff}$.

3) Schmid, Att. 1, 249 f.; 2, 68; 3, 95 f.; Usener, D. heil. Theod. S. 162 f.; vgl. auch die Bemerkung Bergers in Trad. tératol. S. 345 A. 3.

4) $=$ Georgios Monachos p. 18 (Muralt). 
$=$ 'Wenn wir alle in der Welt gleiches Sinnes wären, so würde, da die Welt träge wäre, alles ungeschehen bleiben'.

3. Das Partizip tritt zu seinem Substantiv in einem ganz andern Kasus. Interessant ist hier gleich die erste Stelle, wo nicht weniger als drei Kasus zusammenkommen,

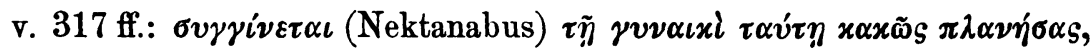

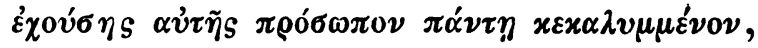

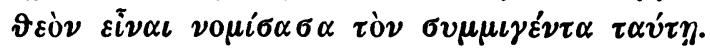

Vgl. ferner 1358.1624 ff. 2526 f. 2654 ff. 2828 ff. 3348 ff. 3783 f. 4470 f.

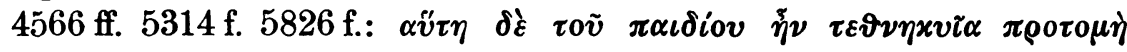
$\mu \dot{\eta} \tau \rho \alpha \varsigma \alpha \dot{v} \tau \tilde{\eta} S \pi \rho \circ x v \dot{\psi} \psi \boldsymbol{v}$, wo das Partizip zu $\pi \alpha \iota \delta i o v$ zu konstruieren ist: 'als es aus dem Mutterleibe herauskam'.

Hier mögen auch die Beispiele Platz finden, wo zu dem absoluten Genetiv ein Dativ oder Accusativ gehört, nämlich: จย์

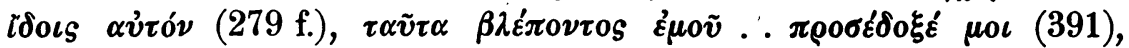
vgl. ferner 525 ff. 1499 ff. 1526 f. 2462 f. 3810 ff. 5847 . 5959 f.

Schliefslich ist zur Charakterisierung der Sprache unseres Verf. noch auf zweierlei hinzuweisen. Einmal liebt er nämlich einzelne Wendungen und Ausdrücke ganz aufserordentlich; so kehrt z. B. die Partikel oṽv oder $\gamma o \tilde{v} \nu$ unaufhörlich wieder, $x \alpha i$ wird sehr häufig angewandt, wo es gar nicht hingehört, Redensarten wie $\nu \tilde{\omega} \tau \alpha \delta \iota \delta \delta \nu \alpha \iota$,

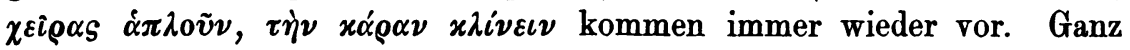

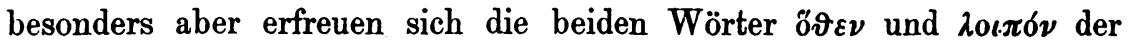
ganz speziellen Vorliebe des Verf. Das erstere hat zwar auch lokale, temporale und kausale Bedeutung, sehr oft aber dient es, ebenso wie $\lambda o \iota \pi \delta \nu$, nur zur Weiterführung und entspricht etwa unserm 'nun'. Im ganzen kommt ö $\vartheta \varepsilon v$ nicht weniger als $113 \mathrm{mal}$ vor, und zwar immer entweder zu Anfang des Verses oder des zweiten Halbverses nach dem Einschnitt (15mal), sonst nie. $\left.{ }^{1}\right)$ Sehr oft wird unmittelbar dahinter, ohne besonderen Grund, xai gesetzt $(36 \mathrm{mal})$, und bisweilen, allerdings

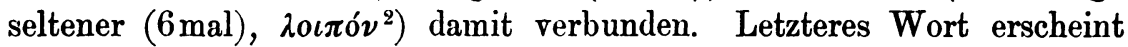
im ganzen $95 \mathrm{mal}$ und dient ebenfalls oft nur als Flickwort. Zweitens zeigt der Verf. eine grofse Freude an zusammengesetzten Verben, ganz hervorragend an solchen mit $x \alpha \tau \alpha \dot{\alpha}$ und $\pi \varrho o ́ s$, sodals im ganzen -

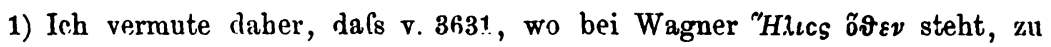

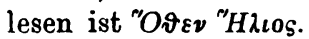

2) Zweimal sind diese beiden Wörter durch andere getrennt, 1732: б̋

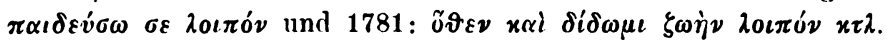




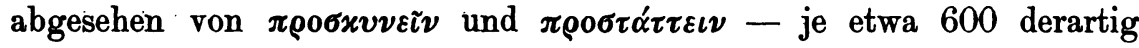
zusammengesetzter Formen vorkommen; dazu kommen dann viele mit

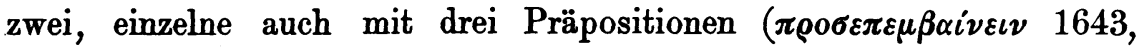
$\pi \rho 0 \sigma \varepsilon \pi \iota x \alpha \vartheta i \zeta \varepsilon \sigma \vartheta \alpha \iota$ 763. 2066, $\alpha \nu \tau \varepsilon \pi \alpha \nu i \sigma \tau \alpha \sigma \vartheta \alpha \iota$ 3279) zusammengesetzte

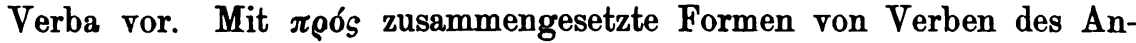
redens erscheinen $114 \mathrm{mal}$, und zwar der Aorist $\pi \rho \circ \varepsilon \varepsilon i \pi o v$ u. s. w. $27 \mathrm{mal}$,

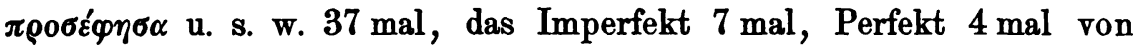

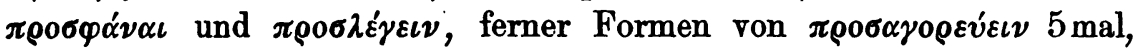
$\pi \rho \circ \sigma \lambda \alpha \lambda \varepsilon i \nu 5 \mathrm{mal}, \pi \rho 0 \sigma \varphi \omega \nu \varepsilon i \nu 26 \mathrm{mal}$, $\pi \rho 0 \sigma \varphi \vartheta \varepsilon \dot{\gamma} \gamma \varepsilon \sigma \vartheta \alpha \iota 3 \mathrm{mal}$, unter welchen die Formen von $\pi \rho 0 \sigma \varepsilon \pi \varepsilon \iota \pi \varepsilon \tilde{\nu}, \pi \rho 0 \sigma \alpha \nu \tau \iota \varphi \eta \tilde{\sigma \alpha \iota, ~ \pi \rho о \sigma \varepsilon \pi \iota \varphi \omega-~}$

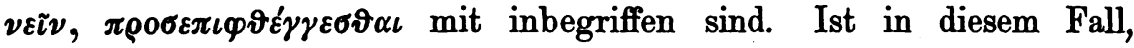
wie in andern, die Präposition im grofsen und ganzen durchaus angebracht, so hat sie in andern vollständig ihre Bedeutung verloren, und das Kompositum entspricht völlig dem einfachen Verbum. Nur einige Beispiele mögen genügen: $\pi \rho \circ \sigma(\xi \nu-, \dot{z} \pi \iota-) \gamma i \gamma \nu \varepsilon \sigma \vartheta \alpha \iota$ kommt $24 \mathrm{mal}$ vor, darunter $19 \mathrm{mal}$ vollständig = yi

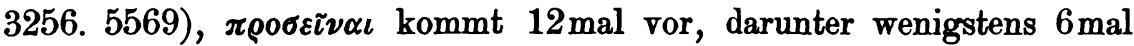

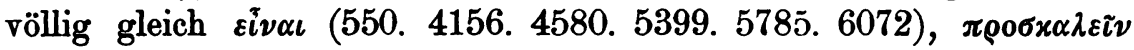
$7 \mathrm{mal}, 4 \mathrm{mal}=$ 'nennen' (4209. 5210. 5281. 5739; ebenso $\pi \varepsilon \rho \iota x \alpha \lambda \varepsilon i \nu ~ 970)$;

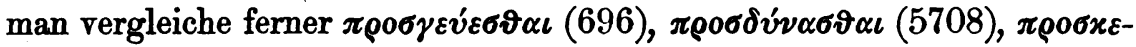

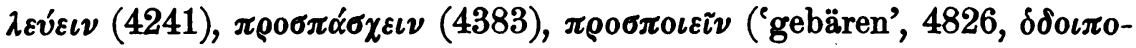

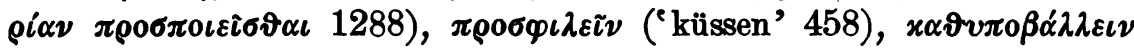

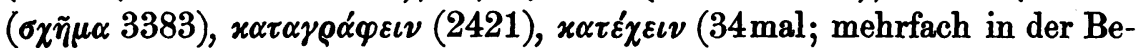
deutung 'haben', z. B. $\pi \alpha \tau \varepsilon$ $\varrho \alpha$ 32, vgl. 703. 1358. 4545 u. s., oder 'halten'

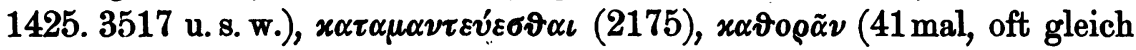

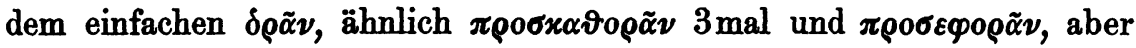

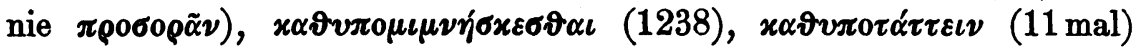

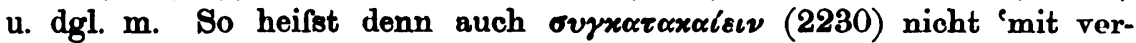

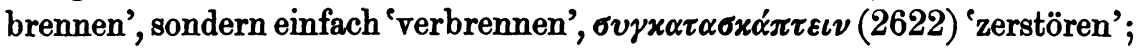

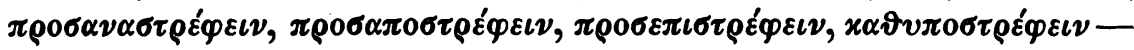

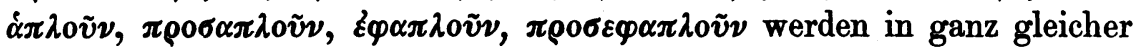
Bedeutung gebraucht; doppelt zusammengesetzte Verba wie $x \alpha \vartheta v \pi \alpha-$

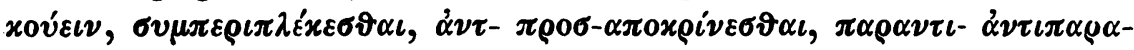
$\tau \alpha^{\alpha} \tau \varepsilon \varepsilon \sigma \alpha \iota, x \alpha \tau \alpha \pi \rho 0 \sigma x v \nu \varepsilon i \nu$ entsprechen dem Sinne nach durchaus den einfach zusammengesetzten Verben. Die wenigen Beispiele werden hoffentlich zur Genüge zeigen, mit welchem Behagen unser Verf. sich derartigen Zusammensetzungen überliels.

Um kurz das Resultat der vorstehenden Auseinandersetzung zu ziehen: unser Verf. ist offenbar ein wissenschaftlich gebildeter Mann 
H. Christensen: Die Sprache des byzantinischen Alexandergedichtes 397

gewesen, der die griechische Sprache auch grammatisch zu erlernen versucht hat. Er hat aber einerseits in mancher Beziehung sich nicht freihalten können von dem Einflufs der lebenden Sprache, wenn sich derselbe auch verhältnismälsig wenig bemerkbar macht, andrerseits ist er durch seine Gelehrsamkeit zu manchen Wunderlichkeiten gekommen, die anfangs das Verständnis manchmal erschweren. Im allgemeinen aber kann ihm das Zeugnis nicht versagt werden, dafs er einfach, gewandt und im grolsen und ganzen ohne allzuviel Pathos, das sich sonst wohl breit macht, schreibt, sodals sich sein Werk leicht und angenehm liest.

Hamburg.

H. Christensen. 